\title{
INDUCED $C^{*}$-ALGEBRAS AND LANDSTAD DUALITY FOR TWISTED COACTIONS
}

\author{
JOHN C. QUIGG AND IAIN RAEBURN
}

\begin{abstract}
Suppose $N$ is a closed normal subgroup of a locally compact group $G$. A coaction $\epsilon: A \rightarrow M\left(A \otimes C^{*}(N)\right)$ of $N$ on a $C^{*}$-algebra $A$ can be inflated to a coaction $\delta$ of $G$ on $A$, and the crossed product $A \times{ }_{\delta} G$ is then isomorphic to the induced $C^{*}$-algebra $\operatorname{Ind}_{N}^{G} A \times_{\epsilon} N$. We prove this and a natural generalization in which $A \times_{\epsilon} N$ is replaced by a twisted crossed product $A \times{ }_{G / N} G$; in case $G$ is abelian, we recover a theorem of Olesen and Pedersen. We then use this to extend the Landstad duality of the first author to twisted crossed products, and give several applications. In particular, we prove that if

$$
1 \rightarrow N \rightarrow G \rightarrow G / N \rightarrow 1
$$

is topologically trivial, but not necessarily split as a group extension, then every twisted crossed product $A \times{ }_{G / N} G$ is isomorphic to a crossed product of the form $A \times N$.
\end{abstract}

\section{INTRODUCTION}

Let $\delta$ be a coaction of a locally compact group $G$ on a $C^{*}$-algebra $B$. The cocrossed product $B \ltimes_{\delta} G$ is a $C^{*}$-algebra whose representations are given by the covariant representations of the cosystem $(B, G, \delta)$; it is generated by a copy $j_{B}(B)$ of $B$ and a copy $j_{G}\left(C_{0}(G)\right)$ of $C_{0}(G)$ (e.g., [LPRS], [Rae3], [Rae4]). The embedding $j_{G}$ is $G$-equivariant, and the first author has recently shown that the existence of such an equivariant embedding characterizes cocrossed products by coactions [Qui2]. In apparently unrelated work, Echterhoff has shown that an ordinary dynamical system $(A, G, \alpha)$ is an induced system $\left(\operatorname{Ind}_{H}^{G} D, G, \tau\right)$ exactly when there is a $G$-equivariant embedding of $C_{0}(G / H)$ in the center of $M(A)$ ([Ech]; for this version of his result, see Section 1). Here we shall show that Echterhoff's theorem does give useful information about cocrossed products by coactions, and especially about the twisted cocrossed products of [PR]. In particular, we use it to extend the theorem of [Qui2] to twisted cocrossed products.

Suppose $\delta$ is a coaction of $G$ on $B$, and $N$ is a closed normal subgroup of $G$ which is amenable. A twist for $\delta$ relative to $G / N$ is a homomorphism $j: C_{0}(G / N) \rightarrow M(B)$ such that $(l, j)$ is a covariant representation of the restricted cosystem $(B, G / N, \delta \mid)$, and $j$ takes values in the fixed-point algebra $M(B)^{\delta}=\{b \in M(B) \mid \delta(b)=b \otimes 1\}$. The twisted crossed product $B \ltimes_{G / N} G$ is the quotient of $B \ltimes_{\delta} G$ whose representations are given by the covariant

Received by the editors January 29, 1994.

1991 Mathematics Subject Classification. Primary 46L55. 
representations $(\pi, \mu):\left(B, C_{0}(G)\right) \rightarrow B(\mathscr{H})$ satisfying $\pi \circ j=\left.\mu\right|_{C_{0}(G / N)}$; it is generated by the image of a covariant representation $\left(k_{B}, k_{G}\right)$ of $(B, G, \delta)$, and the embedding $k_{G}$ of $C_{0}(G)$ is equivariant for the dual $N$-action. Now it is definitely not true that the canonical embeddings $k_{G}$ or $j_{G}$ take values in the center of $M(B \ltimes G)$. However, by convolving $\left.j_{G}\right|_{C_{0}(G / N)}$ with the inverse of the twist $j_{B} \circ j$, we can produce an equivariant embedding of $C_{0}(G / N)$ in $Z M(B \ltimes G)$, and applying Echterhoff's theorem gives an isomorphism of $B \ltimes_{\delta} G$ onto the induced algebra $\operatorname{Ind}_{N}^{G} B \ltimes_{G / N} G$. In the case of abelian $G$, where $\delta$ is given by an action of $\hat{G}$, and $B \ltimes_{G / N} G$ is the twisted covariance algebra $B \rtimes_{N^{\perp}} \hat{G}$ of Green [Gre], we recover a theorem of Olesen and Pedersen [OP3]. Our result indicates why they had to restrict attention to actions of abelian groups: theirs is really a theorem about coactions rather than actions.

We next characterize the twisted cocrossed product $B \ltimes_{G / N} G$ as a $C^{*}$-algebra $A$ with an action $\alpha$ of $N$ (corresponding to the dual action $\hat{\delta}$ ) and an $N$ equivariant embedding of $C_{0}(G)$ (playing the role of $k_{G}$ ). Rather than repeat the whole analysis of [Qui2], we form the induced system $\left(\operatorname{Ind}_{N}^{G} A, G, \tau\right)$, and apply the main theorem of [Qui2] to it, thus realizing Ind $A$ as a cocrossed product $D \ltimes_{\epsilon} G$. We then prove that $\epsilon$ is given by a twist on $G / N$, so that by our previous theorem Ind $A=D \ltimes_{\epsilon} G \cong$ Ind $D \ltimes_{G / N} G$, and deduce from this that $A \cong D \ltimes_{G / N} G$. This does not give the detailed characterization of $B$ as a subalgebra of $M(A)^{\hat{\delta}}$ required for our applications, but we are able to recover this using techniques of Mansfield [Man].

We begin with a section on preliminary matters concerning coactions and the main result of [Qui2]. In Section 2 we discuss the convolution of representations of $C_{0}(G)$, and cocycles for coactions. Section 3 contains the version of Echterhoff's theorem we need. In Section 4 we prove that, if $\delta$ is given by a twist on $G / N$, then $B \ltimes_{\delta} G \cong \operatorname{Ind}_{N}^{G} B \ltimes_{G / N} G$. We use this in Section 5 to characterize twisted cocrossed products (the "Landstad duality"), and in Section 6 we discuss some applications. The most interesting of these-and, in our view, the most surprising-is that, if $G$ is trivial as a principal $N$-bundle, then every twisted cocrossed product $B \ltimes_{G / N} G$ is isomorphic to a cocrossed product $B \ltimes_{\epsilon} N$ by an (untwisted) coaction of $N$. This is analogous to the corresponding property for crossed products by actions of semidirect products (cf. [PR, Section 5(b)]); the surprise is that we only need a topological splitting, rather than an algebraic one.

As in [Qui2] and [PR], we have chosen to work with reduced group algebras and the corresponding reduced coactions. Almost all our results remain true for the full coactions of [Rae3], and this often enables us to lift the hypothesis of amenability from the normal subgroup $N$. In Section 7, we outline the changes that need to be made to carry over our results. The crux of the matter is the link between coactions and representations of $C_{0}(G)$, and we separate a technical aspect of this in an appendix. The second author wishes to point out that the full coactions we study involve full group algebras, but minimal tensor products; the first author has almost convinced him that this theory retains most of the gains over the reduced theory envisaged in [Rae3], while avoiding some of the technical difficulties involved in using maximal tensor products.

This research was carried out while the first author was visiting at the University of Newcastle. He wishes to thank his hosts, particularly Iain Raeburn, 
for their hospitality. The research was supported by the Australian Research Council.

\section{Preliminaries}

Let $G$ be a locally compact group, and let $\lambda_{G}$ and $\rho_{G}$ denote the left and right, respectively, regular representations of $G$ on $L^{2}(G)$. As a bounded strictly continuous $M\left(C_{r}^{*}(G)\right)$-valued function, $\lambda_{G}$ may be regarded as a unitary element of $M\left(C_{0}(G) \otimes C_{r}^{*}(G)\right.$ ) [APT, Corollary 3.4], and we write $W_{G}$ for $\lambda_{G}$ when we have this interpretation in mind. Although it does not matter in this particular case, we mention that we will give all $C^{*}$-tensor products the minimal $C^{*}$-tensor norm.

Definition 1.1. If $A$ is a $C^{*}$-algebra, we say a ${ }^{*}$-subalgebra $Y$ of $M(A)$ is nondegenerate in $M(A)$ if $\overline{Y A}=A$ (where the juxtaposition of two subspaces of an algebra denotes the linear span of the set of products). If $Y$ is a $C^{*}$ subalgebra, then it is nondegenerate in $M(A)$ if and only if some (hence every) bounded approximate identity for $Y$ converges strictly to 1 in $M(A)$. If $B$ is another $C^{*}$-algebra, we say a homomorphism (always assumed *-preserving when between $C^{*}$-algebras) $\pi: A \rightarrow M(B)$ is nondegenerate if its range is nondegenerate in $M(B)$.

Vallin [Val] and Woronowicz [Wor] observe that $C^{*}$-algebras and nondegenerate homomorphisms into multiplier algebras form a category, since a nondegenerate homomorphism of $A$ to $M(B)$ extends uniquely to a strictly continuous (unital) homomorphism of $M(A)$ to $M(B)$, and that the isomorphisms in this category are the usual isomorphisms between $C^{*}$-algebras. For example, the full subcategory of commutative $C^{*}$-algebras is dual to the category of locally compact Hausdorff spaces with continuous maps. More generally (as will come out of the proof of Lemma 3.3), nondegenerate homomorphisms from $C_{0}(X)$ to $Z M(A)$ are in one-to-one correspondence with continuous maps from Prim $A$ to $X$. Another familiar case is given by nondegenerate homomorphisms of $C^{*}(G)$ to $M(A)$, which correspond to strictly continuous unitary representations of $G$ in $M(A)$ (see [Rae1, Proposition 2(2)], for example). A nondegenerate representation of a $C^{*}$-algebra $A$ on a Hilbert space $\mathscr{H}$ may be identified with a nondegenerate homomorphism of $A$ to the multiplier algebra of the $C^{*}$-algebra $\mathscr{K}(\mathscr{H})$ of compact operators on $\mathscr{H}$.

The representation $\lambda_{G} \otimes \lambda_{G}$ determines a nondegenerate homomorphism $\delta_{G}: C_{r}^{*}(G) \rightarrow M\left(C_{r}^{*}(G) \otimes C_{r}^{*}(G)\right)$, and this gives $C_{r}^{*}(G)$ a comultiplication, i.e., $\left(\delta_{G} \otimes l\right) \circ \delta_{G}=\left(l \otimes \delta_{G}\right) \circ \delta_{G}$.

In Sections 1-6 "coaction" will mean "reduced coaction", so that a coaction of $G$ on a $C^{*}$-algebra $B$ is a nondegenerate injective homomorphism $\delta: B \rightarrow$ $M\left(B \otimes C_{r}^{*}(G)\right)$ such that

$$
\delta(B)\left(\mathbf{C} \otimes C_{r}^{*}(G)\right) \subset B \otimes C_{r}^{*}(G) \quad \text { and } \quad(\delta \otimes l) \circ \delta=\left(l \otimes \delta_{G}\right) \circ \delta .
$$

We call the triple $(B, G, \delta)$, or sometimes just the pair $(B, G)$, a cosystem. $\delta$ gives rise to a Banach representation of the reduced Fourier-Stieltjes algebra $B_{r}(G)$ on $B$ via $\delta_{f}=S_{f} \circ \delta$, where $S_{f}$ denotes the slice map determined by $S_{f}(b \otimes c)=f(c) b$ for $f \in B_{r}(G), b \in B$, and $c \in C_{r}^{*}(G)$. Most of the time we only need the restriction of this representation to the Fourier algebra $A(G)$. 
Two cosystems $(B, G, \delta)$ and $(C, G, \epsilon)$ are called conjugate if there is an isomorphism $\theta: B \rightarrow C$ such that $\epsilon \circ \theta=(\theta \otimes l) \circ \delta$, and in this case we also say that $\theta:(B, G) \rightarrow(C, G)$ is a conjugacy.

A cosystem $(B, G, \delta)$, or the coaction $\delta$ itself, is called nondegenerate if $B$ is nondegenerate as an $A(G)$-module. From the context it is always clear whether nondegeneracy of $\delta$ is meant in this latter sense or in the sense of homomorphisms into multiplier algebras. It is an open problem whether every coaction is nondegenerate.

Most of the following result follows from [NT, Theorem A.1]. Recall the subscript notation for placement of tensors, e.g., if $x \in M(A \otimes B)$ then

$$
x_{12}=x \otimes 1 \quad \text { and } \quad x_{13}=\imath \otimes \sigma\left(x_{12}\right),
$$

where $\sigma$ denotes the flip isomorphism determined by $\sigma(a \otimes b)=b \otimes a$. This notation, and obvious adaptations of it, will be used without comment. Recall [LPRS, Remark $3.2(2)$ ] that a unitary $W \in M\left(A \otimes C_{r}^{*}(G)\right)$ is called a corepresentation if it satisfies the corepresentation identity

$$
\imath \otimes \delta_{G}(W)=W_{12} W_{13} .
$$

Lemma 1.2 (Nakagami and Takesaki). The set of nondegenerate homomorphisms $\mu: C_{0}(G) \rightarrow M(A)$ is in one-to-one correspondence with the set of corepresentations $W \in M\left(A \otimes C_{r}^{*}(G)\right)$. The correspondence is determined by

$$
\begin{gathered}
W=\mu \otimes \imath\left(W_{G}\right), \\
\mu(f)=S_{f}(W), \quad f \in A(G),
\end{gathered}
$$

and we write $W_{\mu}$ for $\mu \otimes \imath\left(W_{G}\right)$.

Proof. The only new assertion here is that if $W \in M\left(A \otimes C_{r}^{*}(G)\right)$ is a corepresentation, then the corresponding homomorphism $\mu: C_{0}(G) \rightarrow M(A)$ is nondegenerate. We verify this here, borrowing an approximation argument from [LPRS, Theorem 2.9]. Let $a \in A$, and choose $f \in A(G)$ with $f(e)=1$. Then

$$
\begin{aligned}
a & =a f(e)=a S_{f}(1)=S_{f}(a \otimes 1) \\
& =S_{g \cdot x}(a \otimes 1), \quad \text { for some } g \in A(G), x \in C_{r}^{*}(G) \\
& =S_{g}\left((a \otimes x) W^{*} W\right) \\
& \approx \sum_{i} S_{g}\left(\left(a_{i} \otimes x_{i}\right) W\right), \quad \text { for finitely many } a_{i} \in A, x_{i} \in C_{r}^{*}(G) \\
& =\sum_{i} a_{i} \mu\left(g \cdot x_{i}\right) .
\end{aligned}
$$

Hence, $A \subset \overline{A \mu\left(C_{0}(G)\right)}$, showing that $\mu$ is nondegenerate.

We can use a nondegenerate homomorphism $\mu: C_{0}(G) \rightarrow M(A)$ to define a coaction $\delta$ of $G$ on $A$ by

$$
\delta(a)=\operatorname{Ad} W_{\mu}(a \otimes 1),
$$

and as in [LPRS, Definition 2.7] we call such a coaction unitary. More generally, if $\delta$ restricts to give a coaction, still denoted by $\delta$, on a $C^{*}$-subalgebra $B$ of $M(A)$, we say the cosystem $(B, G, \delta)$ is implemented by $\mu$, and write $\delta=\operatorname{Ad} W_{\mu}$. 
We extend the concept of covariant representations slightly: let a $(B, G, \delta)$ be a cosystem. A covariant representation of $(B, G, \delta)$ in $M(A)$ will mean a pair $(\pi, \mu)$, where $\pi: B \rightarrow M(A)$ and $\mu: C_{0}(G) \rightarrow M(A)$ are nondegenerate homomorphisms satisfying the covariance identity $(\pi \otimes l) \circ \delta=\operatorname{Ad} W_{\mu} \circ(\pi \otimes 1)$. Of course, a covariant representation in the usual sense on a Hilbert space $\mathscr{H}$ can be identified with an covariant representation in $M(\mathscr{K}(\mathscr{H}))$, and a covariant representation in $M(A)$ can be made into a covariant representation on a Hilbert space by composing with any faithful, nondegenerate representation of $A$. The discussion of [Qui2, Section 2] concerning covariant representations carries over almost verbatim to the present context. In particular, if $(\pi, \mu)$ is a covariant representation of $(B, G, \delta)$ in $M(A)$ then $C^{*}(\pi, \mu)=$ $\overline{\pi(B) \mu\left(C_{0}(G)\right)}$ is a $C^{*}$-subalgebra of $M(A)$. Moreover, Ad $W_{\mu}$ gives a coaction of $G$ on $\pi(B)$, nondegenerate if $\delta$ is, and when $\pi$ is faithful it implements a conjugacy between the cosystems $(B, G, \delta)$ and $\left(\pi(B), G, \operatorname{Ad} W_{\mu}\right)$.

Let $(\pi, \mu)$ be a covariant representation of a cosystem $(B, G, \delta)$. We say that $\left(C^{*}(\pi, \mu), \pi, \mu\right)$ is a cocrossed product of the cosystem $(B, G, \delta)$ if every covariant representation $(\rho, \nu)$ factors through $(\pi, \mu)$, i.e., if there is a homomorphism $\theta$ of $C^{*}(\pi, \mu)$ such that $\theta \circ \pi=\rho$ and $\theta \circ \mu=\nu$. As usual, up to isomorphism there is a unique cocrossed product, and we denote a generic one by $\left(B \ltimes_{\delta} G, j_{B}^{\delta}, j_{G}^{\delta}\right)$. We write $\left(B \ltimes G, j_{B}, j_{G}\right)$ if $\delta$ is understood, and for a covariant representation $(\pi, \mu)$ of $(B, G)$ we let $\pi \times \mu$ denote the (unique) homomorphism of $B \times G$ such that $(\pi \times \mu) \circ j_{B}=\pi$ and $(\pi \times \mu) \circ j_{G}=\mu$. When we say that a $C^{*}$-algebra $A$ is isomorphic to $B \ltimes G$, we mean that there is a covariant representation $(\pi, \mu)$ of $(B, G, \delta)$ such that $A=C^{*}(\pi, \mu)$ and $(A, \pi, \mu)$ is a cocrossed product of $(B, G, \delta)$. If $\pi$ is a nondegenerate homomorphism of $B$ in $M(A)$, then Ind $\pi=((\pi \otimes l) \circ \delta, 1 \otimes l)$ is a covariant representation of $(B, G, \delta)$ in $M\left(A \otimes \mathscr{K}\left(L^{2}(G)\right)\right)$, called the regular covariant representation induced by $\pi$, and when $\pi$ is faithful $\left(C^{*}(\operatorname{Ind} \pi)\right.$, Ind $\left.\pi\right)$ is a cocrossed product of $(B, G, \delta)$ [LPRS, Theorem 3.7]. The dual action $\hat{\delta}$ of $G$ on $B \ltimes_{\delta} G$ is determined by

$$
\hat{\delta}_{s}\left(j_{B}(b) j_{G}(f)\right)=j_{B}(b) j_{G}(s \cdot f), \quad s \in G .
$$

Here and in the sequel we use the $G$-actions on $C_{0}(G)$ given by

$$
(s \cdot f)(t)=f(t s) \text { and }(f \cdot s)(t)=f(s t),
$$

which we term right and left translation, respectively. Katayama's duality theorem [Kat, Theorem 8] states that if $\delta$ is nondegenerate then

$$
B \ltimes_{\delta} G \rtimes_{\hat{\delta}, r} G=B \otimes \mathscr{K}\left(L^{2}(G)\right),
$$

where the subscript $r$ indicates the reduced crossed product.

The terminology involving actions, systems, crossed products, etc., is exactly analogous to that which we have introduced for coactions; in particular, we denote the crossed product of a system $(A, G)$ by $A \rtimes G$.

For later convenience, we record the following elementary

Proposition 1.3. If $\theta:(B, G, \delta) \rightarrow(C, G, \epsilon)$ is a conjugacy between cosystems, then there is a conjugacy $\Phi:\left(B \ltimes_{\delta} G, G\right) \rightarrow\left(C \ltimes_{\epsilon} G, G\right)$ such that

$$
\Phi \circ j_{B}=j_{C} \circ \theta \text { and } \Phi \circ j_{G}^{\delta}=j_{G}^{\epsilon} \text {. }
$$


Proof. It is straightforward to check that $\left(j_{C} \circ \theta, j_{G}^{\epsilon}\right)$ is a covariant representation of $(B, G, \delta)$ in $M\left(C \ltimes_{\epsilon} G\right)$ and that the corresponding nondegenerate homomorphism $\Phi=\left(j_{C} \circ \theta\right) \times j_{G}^{\epsilon}: B \ltimes_{\delta} G \rightarrow M\left(C \ltimes_{\epsilon} G\right)$ is $G$-equivariant with range $C \ltimes_{\epsilon} G$. It follows from [Qui2, Proposition 3.1] that $\Phi$ is an isomorphism. Moreover, $\Phi \circ j_{B}=j_{C} \circ \theta$ and $\Phi \circ j_{G}^{\delta}=j_{G}^{\epsilon}$, so $\Phi$ conjugates $\left(B \ltimes_{\delta} G, G\right)$ to $\left(C \ltimes_{\epsilon} G, G\right)$.

We will need the following adaptation of the construction from [Qui2, Definition 3.4-Definition 3.9], which is based upon work of Olesen and Pedersen [OP1, Section 2], [OP2, note added in proof]. Let $(A, G)$ be a system, and define the sets

$$
\left.\begin{array}{rl}
\mathfrak{p}_{G}(A) & =\left\{a \in M(A)^{+} \mid \begin{array}{l}
\text { there exists } E_{G}^{A} a \in M(A)^{+} \text {with } \\
\left\langle E_{G}^{A} a, \phi\right\rangle=\int_{G}\langle s \cdot a, \phi\rangle d s \text { for all } \phi \in A^{*+}
\end{array}\right.
\end{array}\right\},
$$

We frequently omit parts of the notation. By [Qui2, Lemma 3.5 and Corollary 3.6], $\mathfrak{m}$ is a selfadjoint subalgebra of $M(A)$ with positive part $\mathfrak{p}, E$ extends uniquely to a positive linear map from $\mathfrak{m}$ into $M(A)$ such that

$$
E a=\int_{G} s \cdot a d s \quad \text { for all } a \in \mathfrak{m},
$$

where the integral is taken in the weak ${ }^{*}$ topology of $A^{* *}$, and the map $a \mapsto$ $E(b a c)$ is norm continuous on $M(A)$ for any $b, c \in \mathfrak{m}$. When a closed subgroup $H$ of $G$ acts by right translation on $C_{0}(G)$, one readily checks that

$$
C_{c}(G) \subset \mathfrak{m}_{H}\left(C_{0}(G)\right)
$$

and

$$
\left(E_{H}^{C_{0}(G)} f\right)(s)=\int_{H} f(s h) d h, \quad f \in C_{c}(G), s \in G .
$$

Proposition 1.4. If $G$ acts on $C^{*}$-algebras $A$ and $B$, and if $\pi: A \rightarrow M(B)$ is a G-equivariant nondegenerate homomorphism, then $\pi(\mathfrak{m}(A)) \subset \mathfrak{m}(B)$, and $\pi \circ E^{A}=E^{B} \circ \pi$ on $\mathfrak{m}(A)$.

Proof. For $a \in \mathfrak{p}(A)$ and $\omega \in B^{*+}$ we have

$$
\begin{aligned}
\int_{G}\langle t \cdot \pi(a), \omega\rangle d t & =\int_{G}\langle\pi(t \cdot a), \omega\rangle d t=\int_{G}\left\langle t \cdot a, \pi^{*}(\omega)\right\rangle d t \\
& =\left\langle E^{A} a, \pi^{*}(\omega)\right\rangle=\left\langle\pi \circ E^{A}(a), \omega\right\rangle,
\end{aligned}
$$

so we have $\pi(a) \in \mathfrak{p}(B)$ and

$$
\pi \circ E^{A}(a)=E^{B} \circ \pi(a) .
$$

Since $\pi$ is a ${ }^{*}$-homomorphism, the result follows.

Definition 1.5. If $(A, G)$ is a system, $X$ is a *-subalgebra of $M(A)$, and $Y$ is $a^{*}$-subalgebra of $\mathfrak{m}(A)$, let

$$
\operatorname{Fix}(X, G, Y)=C^{*}(E(Y X Y)) .
$$

If $(B, G)$ is another system, and if $\mu: B \rightarrow M(A)$ is a $G$-equivariant nondegenerate homomorphism, let

$$
\operatorname{Fix}(X, G, \mu)=\operatorname{Fix}(X, G, \mu(\mathfrak{m}(B))) .
$$


When we apply the above construction, we will almost always take $X$ to be $A$. Landstad duality for coactions [Qui2, Theorem 3.3] states that a system $(A, G)$ is of the form $(B \ltimes G, G)$ for some cosystem $(B, G)$ if and only if there is a $G$-equivariant nondegenerate homomorphism $\mu: C_{0}(G) \rightarrow A$, and in this case $B$ may be taken to be $\operatorname{Fix}(A, G, \mu)$.

Lemma 1.6. If $(A, G)$ is a system and $Y$ and $Z$ are commuting *-subalgebras of $\mathfrak{m}(A)$ which are nondegenerate in $M(A)$, then

$$
\operatorname{Fix}(A, G, Y)=\operatorname{Fix}(A, G, Z) .
$$

In particular, if $G$ is a closed subgroup of a locally compact group $L$, and if $\mu: C_{0}(L) \rightarrow M(A)$ is a G-equivariant nondegenerate homomorphism, then $\operatorname{Fix}(A, G, \mu)=\operatorname{Fix}\left(A, G, \mu\left(C_{c}(L)\right)\right)$.

Proof. For $y_{1}, y_{2} \in Y, z_{1}, z_{2} \in Z$, and $a \in A$ we have $E\left(y_{1} z_{1} a z_{2} y_{2}\right)=$ $E\left(z_{1} y_{1} a y_{2} z_{2}\right)$, so $\operatorname{Fix}(A, G, Y) \subset \operatorname{Fix}(A, G, Z)$ by nondegeneracy of $Z$ and continuity of $a \mapsto E\left(y_{1} a y_{2}\right)$, and the result follows by symmetry.

Lemma 1.7. Let $(A, G)$ be a system, $Y$ a nondegenerate ${ }^{*}$-subalgebra of $\mathfrak{m}(A)$, and $C$ a $C^{*}$-subalgebra of $M(A)^{G}$ (the set of $G$-fixed elements of $M(A)$ ) which is nondegenerate in $M(A)$. If $C$ commutes with $Y$, then $C$ is also a nondegenerate $C^{*}$-subalgebra of $M(\operatorname{Fix}(A, G, Y))$.

Proof. To see that $C \subset M(\operatorname{Fix}(A, G, Y))$, it is enough to observe that for $c \in$ $C, y, z \in Y$, and $a \in A$ we have $c E(y a z)=E(y c a z) \in \operatorname{Fix}(A, G, Y)$, and similarly for $E(y a z) c$. Moreover, if $\left\{e_{i}\right\}$ is a bounded approximate identity for $C$, then

$$
e_{i} E(y a z)=E\left(y e_{i} a z\right) \rightarrow E(y a z),
$$

since $C$ is nondegenerate in $M(A)$ and $a \mapsto E(y a z)$ is continuous. Therefore, $C$ is nondegenerate in $M(\operatorname{Fix}(A, G, Y))$.

Corollary 1.8. Let $(A, G)$ be a system and $Y$ a nondegenerate *-subalgebra of $\mathfrak{m}_{G}(A) \cap Z M(A)$, where $Z M(A)$ denotes the center of $M(A)$. Then

$$
M(\operatorname{Fix}(A, G, Y))=M(A)^{G} .
$$

Proof. $M(A)^{G} \subset M(\operatorname{Fix}(A, G, Y))$ by Lemma 1.7. The opposite containment holds even without centrality of $Y$.

\section{CONVOLUTIONS AND COCYCLES}

The following concept, which will be crucial in the sequel, generalizes [NT, Definition A.2]. Suppose $\mu, \nu: C_{0}(G) \rightarrow M(A)$ are commuting nondegenerate homomorphisms. There is a nondegenerate homomorphism $\mu \times \nu: C_{0}(G) \otimes$ $C_{0}(G) \rightarrow M(A)$ such that

$$
\mu \times \nu(f \otimes g)=\mu(f) \nu(g) .
$$

We let $\alpha^{G}: C_{0}(G) \rightarrow M\left(C_{0}(G) \otimes C_{0}(G)\right)$ be the nondegenerate homomorphism defined by

$$
\alpha^{G}(f)(s, t)=f(s t)
$$


Definition 2.1. Let $\mu, \nu: C_{0}(G) \rightarrow M(A)$ be commuting nondegenerate homomorphisms. The convolution of $\mu$ and $\nu$ is the nondegenerate homomorphism

$$
\mu * \nu=(\mu \times \nu) \circ \alpha^{G}
$$

of $C_{0}(G)$ to $M(A)$.

Nakagami and Takesaki defined the special case where $\mu$ and $\nu$ are of the form $\mu_{0} \otimes 1$ and $1 \otimes \nu_{0}$, respectively. Proposition 2.2 and Corollary 2.5 below generalize [NT, Theorem A.3] to our context.

Proposition 2.2. If $\mu$ and $\nu$ are commuting nondegenerate homomorphisms of $C_{0}(G)$ to $M(A)$, then

$$
W_{\mu * \nu}=W_{\mu} W_{\nu} .
$$

Proof. We just have to apply $(\mu \times \nu) \otimes l$ to the identity $\alpha^{G} \otimes l\left(W_{G}\right)=$ $\left(W_{G}\right)_{13}\left(W_{G}\right)_{23}$.

Proposition 2.3. Any maximal commuting set of nondegenerate homomorphisms from $C_{0}(G)$ to $M(A)$ is a group with convolution as product, identity element given by the trivial nondegenerate homomorphism $\mu_{e}: f \mapsto f(e) 1$, and the inverse $\mu^{\vee}$ of $\mu$ given by $\mu^{\vee}(f)=\mu\left(f^{\vee}\right)$, where $f^{\vee}(s)=f\left(s^{-1}\right)$.

Proof. This follows from Proposition 2.2 and the formulae

$$
W_{\mu_{e}}=1, \quad W_{\mu^{\vee}}=W_{\mu}^{*} .
$$

We will need another formula involving $\mu * \nu$, and we pave the way with an integral formula for $\alpha^{G}$ :

Proposition 2.4. For $f, g \in C_{c}(G)$ we have

$$
\alpha^{G}(f * g)=\int_{G} t \cdot f \otimes g \cdot t^{-1} d t,
$$

the integral converging in the weak ${ }^{*}$ topology of $\left(C_{0}(G) \otimes C_{0}(G)\right)^{* *}$.

Proof. For $\mu \in M(G \times G)$ we have

$$
\begin{aligned}
\int \alpha^{G}(f * g) d \mu & =\iint \alpha^{G}(f * g)(r, s) d \mu(r, s) \\
& =\iiint f(t) g\left(t^{-1} r s\right) d t d \mu(r, s) \\
& =\iiint f(r t) g\left(t^{-1} s\right) d t d \mu(r, s) \\
& =\iiint t \cdot f(r) g \cdot t^{-1}(s) d \mu(r, s) d t \\
& =\iint t \cdot f \otimes g \cdot t^{-1} d \mu d t .
\end{aligned}
$$

Corollary 2.5. If $\mu, \nu: C_{0}(G) \rightarrow M(A)$ are commuting nondegenerate homomorphisms, then for $f, g \in C_{c}(G)$ we have

$$
\mu * \nu(f * g)=\int \mu(t \cdot f) \nu\left(g \cdot t^{-1}\right) d t,
$$


the integral converging in the weak ${ }^{*}$ topology of $A^{* *}$.

Proof. For $\omega \in A^{*}$ we have

$$
\begin{aligned}
\langle\mu * \nu(f * g), \omega\rangle & =\left\langle(\mu \times \nu) \circ \alpha^{G}(f * g), \omega\right\rangle \\
& =\left\langle\alpha^{G}(f * g),(\mu \times \nu)^{*}(\omega)\right\rangle \\
& =\int\left\langle t \cdot f \otimes g \cdot t^{-1},(\mu \times \nu)^{*}(\omega)\right\rangle d t \\
& =\int\left\langle\mu \times \nu\left(t \cdot f \otimes g \cdot t^{-1}\right), \omega\right\rangle d t \\
& =\int\left\langle\mu(t \cdot f) \nu\left(g \cdot t^{-1}\right), \omega\right\rangle d t .
\end{aligned}
$$

Let $N$ be an amenable closed normal subgroup of $G$, and let $\lambda_{G, N}: C_{r}^{*}(G) \rightarrow$ $C_{r}^{*}(G / N)$ denote the natural quotient map. We often identify $C_{0}(G / N)$, $A(G / N)$, etc. with algebras of functions on $G$ which are constant on $N$-cosets. If $\mu$ is a homomorphism of $C_{0}(G)$ we sometimes denote the restriction of $\mu$ to $C_{0}(G / N)$ by $\mu$.

Lemma 2.6. If $\mu$ and $\nu$ are commuting nondegenerate homomorphisms of $C_{0}(G)$, then $(\mu * \nu)|=\mu| * \nu \mid$.

Proof. Since $W_{G / N}=\imath \otimes \lambda_{G, N}\left(W_{G}\right)$, we have

$$
\begin{aligned}
W_{(\mu * \nu) \mid} & =\imath \otimes \lambda_{G, N}\left(W_{\mu * \nu}\right)=\imath \otimes \lambda_{G, N}\left(W_{\mu} W_{\nu}\right) \\
& =\imath \otimes \lambda_{G, N}\left(W_{\mu}\right) \imath \otimes \lambda_{G, N}\left(W_{\nu}\right)=W_{\mu \mid} W_{\nu \mid} .
\end{aligned}
$$

As we have already mentioned, [Qui2, Theorem 3.3] says that a system $(A, G)$ is of the form $(B \ltimes G, G)$ if and only if there is a $G$-equivariant nondegenerate homomorphism $\mu: C_{0}(G) \rightarrow M(A)$, and in this case $B$ may be taken to be $\operatorname{Fix}(A, G, \mu)$. Moreover, with this choice the cosystem $(B, G)$ is nondegenerate, and its conjugacy class among nondegenerate cosystems with dual system $(A, G)$ is uniquely determined by the further requirement that $j_{G}=\mu$. So, a fixed $G$-equivariant nondegenerate homomorphism $\mu$ determines a conjugacy class of cosystems $(B, G)$. What if we allow $\mu$ to vary? As the following discussion will show, it is tempting to guess that we should get an outer conjugacy class of cosystems.

The next definition expands upon [LPRS, Definition 2.7].

Definition 2.7. (i) A cocycle for a cosystem $(B, G, \delta)$ (or a $\delta$-cocycle) is a unitary $U \in M\left(B \otimes C_{r}^{*}(G)\right)$ satisfying

$$
\begin{gathered}
l \otimes \delta_{G}(U)=U_{12} \delta \otimes l(U) ; \\
\operatorname{Ad} U \circ \delta(B)\left(1 \otimes C_{r}^{*}(G)\right) \subset B \otimes C_{r}^{*}(G) .
\end{gathered}
$$

(ii) If $U$ is a $\delta$-cocycle, then $\operatorname{Ad} U \circ \delta$ is a coaction of $G$ on $B$, which we call exterior equivalent to $\delta$.

(iii) Cosystems $(B, G, \delta)$ and $(C, G, \epsilon)$ are called outer conjugate if there is a $\delta$-cocycle $U$ such that $\operatorname{Ad} U \circ \delta$ is conjugate to $\epsilon$.

We call (2.1) the cocycle identity. We do not know whether (2.2) is redundant. If $U$ is a $\delta$-cocycle and $\epsilon=\operatorname{Ad} U \circ \delta$, then $U^{*}$ is an $\epsilon$-cocycle and $\delta=$ Ad $U^{*} \circ \epsilon$, and if moreover $V$ is an $\epsilon$-cocycle and $\gamma=\operatorname{Ad} V \circ \epsilon$, then $V U$ is 
a $\delta$-cocycle and $\gamma=\operatorname{Ad} V U \circ \delta$. Hence, exterior equivalence is an equivalence relation. Lemma 1.2 says that a cocycle for the trivial coaction of $G$ on $B$ is just a nondegenerate homomorphism of $C_{0}(G)$ to $M(B)$ (and here (2.2) is redundant). Moreover, unitary coactions are precisely those which are exterior equivalent to trivial coactions.

Part (i) of the following result is a slight extension of [LPRS, Theorem 2.9], which did not mention the dual action.

Proposition 2.8. (i) Let $(B, G, \delta)$ and $(B, G, \epsilon)$ be exterior equivalent cosystems. Then there is a conjugacy $\Phi:\left(B \ltimes_{\delta} G, G\right) \rightarrow\left(B \ltimes_{\epsilon} G, G\right)$ such that

$$
\Phi \circ j_{B}^{\delta}=j_{B}^{\epsilon} \text {. }
$$

(ii) Let $(B, G, \delta)$ and $(C, G, \epsilon)$ be outer conjugate cosystems. Then there are a conjugacy $\Phi:\left(B \ltimes_{\delta} G, G\right) \rightarrow\left(C \ltimes_{\epsilon} G, G\right)$ and an isomorphism $\theta: B \rightarrow C$ such that

$$
\Phi \circ j_{B}=j_{C} \circ \theta \text {. }
$$

Proof. For (i), let $U$ be a $\delta$-cocycle such that $\epsilon=\operatorname{Ad} U \circ \delta$. We first construct a bijection between the covariant representations of the cosystems $(B, G, \delta)$ and $(B, G, \epsilon)$. Let $(\pi, \mu)$ be a covariant representation of $(B, G, \delta)$, and define

$$
V=\pi \otimes l(U) W_{\mu} .
$$

Clearly $V$ is a unitary element of $M\left(C^{*}(\pi, \mu) \otimes C_{r}^{*}(G)\right)$. The corepresentation identity is a straightforward calculation, e.g., [LPRS, Theorem 2.9]. Hence, by Lemma 1.2 there is a unique nondegenerate homomorphism $\nu: C_{0}(G) \rightarrow$ $M\left(C^{*}(\pi, \mu)\right)$ such that $V=W_{\nu}$.

We next claim that $(\pi, \nu)$ is a covariant representation of $(B, G, \epsilon)$ in $M\left(C^{*}(\pi, \mu)\right)$. We need only check the covariance identity: for $b \in B$ we have

$$
\begin{aligned}
(\pi \otimes l) \circ \epsilon(b) & =(\pi \otimes \imath) \circ \operatorname{Ad} U \circ \delta(b) \\
& =\operatorname{Ad}(\pi \otimes \imath(U)) \circ(\pi \otimes \imath) \circ \delta(b) \\
& =\operatorname{Ad}(\pi \otimes \imath(U)) \circ \operatorname{Ad} W_{\mu}(\pi(b) \otimes 1) \\
& =\operatorname{Ad} W_{\nu}(\pi(b) \otimes 1) .
\end{aligned}
$$

We now have a mapping $(\pi, \mu) \mapsto(\pi, \nu)$ of covariant representations of $(B, G, \delta)$ to those of $(B, G, \epsilon)$. Since we can recover $\mu$ from $\nu$ via the $\epsilon$-cocycle $U^{*}$, the mapping $(\pi, \mu) \mapsto(\pi, \nu)$ is a bijection.

We will need to know that $C^{*}(\pi, \mu)=C^{*}(\pi, \nu)$. By symmetry it suffices to show that $C^{*}(\pi, \mu) \supset C^{*}(\pi, \nu)$. An approximation argument similar to the proof of Lemma 1.2 shows that for $b \in B$ and $f \in A(G)$ we have

$\pi(b) \nu(f) \approx \sum_{i} \pi\left(b_{i}\right) \mu\left(g \cdot x_{i}\right) \quad$ for some $b_{i} \in B, g \in A(G)$, and $x_{i} \in C_{r}^{*}(G)$.

Hence $\pi(b) \nu(f) \in C^{*}(\pi, \mu)$, which gives $C^{*}(\pi, \mu) \supset C^{*}(\pi, \nu)$ since $A(G)$ is dense in $C_{0}(G)$.

We will now show that $\pi \times \mu$ is faithful if and only if $\pi \times \nu$ is, and again by symmetry it suffices to show that fidelity of $\pi \times \nu$ follows from that of $\pi \times \mu$. Let $\left(\pi^{\prime}, \nu^{\prime}\right)$ be another covariant representation of $(B, G, \epsilon)$, and let $\left(\pi^{\prime}, \mu^{\prime}\right)$ be the corresponding covariant representation of $(B, G, \delta)$ as above. Assuming that $\pi \times \mu$ is faithful, there is a nondegenerate homomorphism $\phi$ of 
$C^{*}(\pi, \mu)$ such that $\pi^{\prime}=\phi \circ \pi$ and $\mu^{\prime}=\phi \circ \mu$. We may also regard $\phi$ as a homomorphism of $C^{*}(\pi, \nu)$. We have

$$
W_{\nu^{\prime}}=\pi^{\prime} \otimes l(U) W_{\mu^{\prime}}=\phi \otimes l\left(\pi \otimes l(U) W_{\mu}\right)=\phi \otimes l\left(W_{\nu}\right) .
$$

Hence $\nu^{\prime}=\phi \circ \nu$, and we have shown that $\pi \times \nu$ is faithful.

We see from the preceding arguments that $\left(B \ltimes_{\delta} G, j_{B}^{\delta}, \nu\right)$ is a cocrossed product for $(B, G, \epsilon)$, where $W_{\nu}=j_{B}^{\delta} \otimes l(U) j_{G}^{\delta} \otimes l\left(W_{G}\right)$. Hence, the identity map of $B \ltimes_{\delta} G$ may be regarded as an isomorphism $\Phi: B \ltimes_{\delta} G \rightarrow B \ltimes_{\epsilon} G$ with $\Phi \circ j_{B}^{\delta}=j_{B}^{\epsilon}$.

To finish the proof of (i), we must show that $\Phi$ intertwines the dual actions $\hat{\delta}$ and $\hat{\epsilon}$, i.e., $\hat{\delta}$ and $\hat{\epsilon}$ coincide. Fix $s \in G$. Since $j_{B}^{\delta}=j_{B}^{\epsilon}$, it suffices to show that $\hat{\delta}_{s} \circ j_{G}^{\epsilon}(f)=j_{G}^{\epsilon}(s \cdot f)$ for all $f \in C_{0}(G)$, and this is an immediate consequence of the following calculation:

$$
\begin{aligned}
\hat{\delta}_{s} \circ j_{G}^{\epsilon} \otimes l\left(W_{G}\right) & =\hat{\delta}_{s} \otimes \imath\left(j_{B}^{\delta} \otimes \imath(U) j_{G}^{\delta} \otimes \imath\left(W_{G}\right)\right) \\
& =j_{B}^{\delta} \otimes \imath(U)\left(\hat{\delta}_{s} \circ j_{G}^{\delta}\right) \otimes \imath\left(W_{G}\right) \\
& =j_{B}^{\delta} \otimes \imath(U) j_{G}^{\delta} \otimes \imath\left(W_{G}\left(1 \otimes \lambda_{G}(s)\right)\right) \\
& =j_{B}^{\delta} \otimes \imath(U) j_{G}^{\delta} \otimes \imath\left(W_{G}\right)\left(1 \otimes \lambda_{G}(s)\right) \\
& =j_{G}^{\epsilon} \otimes \imath\left(W_{G}\right)\left(1 \otimes \lambda_{G}(s)\right) \\
& =j_{G}^{\epsilon} \otimes \imath\left(W_{G}\left(1 \otimes \lambda_{G}(s)\right)\right) .
\end{aligned}
$$

(ii) follows from (i) and Proposition 1.3.

When $G$ is abelian, coactions of $G$ correspond to actions of $\hat{G}$, and the converses of both parts of Proposition 2.8 are true, by a result of Pedersen [Ped], amplified by [RR, Theorem 0.10$]$. The converse of part (ii) would follow from that of part (i). To see this, let $\Phi:\left(B \ltimes_{\delta} G, G\right) \rightarrow\left(C \ltimes_{\epsilon} G, G\right)$ be a conjugacy and $\theta: B \rightarrow C$ an isomorphism such that $\Phi \circ j_{B}^{\delta}=j_{C}^{\epsilon} \circ \theta$. Then $\gamma=\left(\theta^{-1} \otimes l\right) \circ \epsilon \circ \theta$ is a coaction of $G$ on $B$ which is conjugate to $\epsilon$ via $\theta^{-1}$. By Proposition 1.3 there is a conjugacy $\Psi:\left(C \ltimes_{\epsilon} G, G\right) \rightarrow\left(B \ltimes_{\gamma} G, G\right)$ such that $\Phi \circ j_{C}^{\epsilon}=j_{B}^{\gamma} \circ \theta^{-1}$. But then $\Psi \circ \Phi:\left(B \ltimes_{\delta} G, G\right) \rightarrow\left(B \ltimes_{\gamma} G, G\right)$ is a conjugacy, and

$$
\Psi \circ \Phi \circ j_{B}^{\delta}=\Psi \circ j_{C}^{\epsilon} \circ \theta=j_{B}^{\gamma} .
$$

Hence, if the converse of Proposition 2.8 (i) holds, then $\delta$ and $\gamma$ are exterior equivalent, so $\delta$ and $\epsilon$ are outer conjugate. We conjecture that this converse does in fact hold. Let us reformulate this slightly. If $\epsilon$ is a coaction of $G$ on $B$ which is exterior equivalent to $\delta$, applying the proof of Proposition 2.8 (i) to $\left(j_{B}^{\delta}, j_{G}^{\delta}\right)$ gives a $G$-equivariant nondegenerate homomorphism of $C_{0}(G)$ into $M\left(B \ltimes_{\delta} G\right)$ implementing a coaction on $j_{B}^{\delta}(B)$ which is conjugate to $\epsilon$ via $j_{B}^{\delta}$. We conjecture that, conversely, if there is a $G$-equivariant nondegenerate homomorphism implementing a coaction $\gamma$ on $j_{B}^{\delta}(B)$, then the coaction $\left(j_{B}^{-1} \otimes\right.$ $l) \circ \gamma^{\circ} j_{B}$ is exterior equivalent to $\delta$. However, we can get by with the following, which we will generalize to the context of twisted coactions in Section 6 .

Proposition 2.9. Let $(A, G)$ be a system, and let $\mu, \nu: C_{0}(G) \rightarrow M(A)$ be commuting G-equivariant nondegenerate homomorphisms. Then $\operatorname{Fix}(A, G, \mu)=$ $\operatorname{Fix}(A, G, \nu)$, and the G-coactions (vouchsafed by [Qui2, Theorem 3.3]) Ad $W_{\mu}$ 
and $\operatorname{Ad} W_{\nu}$ on this common $C^{*}$-algebra are exterior equivalent via the $\operatorname{Ad} W_{\mu}$ cocycle $W_{\nu * \mu}$.

Proof. The first statement is immediate from Lemma 1.6. A calculation using Corollary 2.5 shows that the nondegenerate homomorphism $\nu * \mu^{\vee}: C_{0}(G) \rightarrow$ $M(A)$ commutes with $\mu$ and takes values in $M(A)^{G}$, hence is a nondegenerate homomorphism to $M(\operatorname{Fix}(A, G, \mu))$ by Lemma 1.7. Therefore, $W_{\nu * \mu} \vee \in$ $M\left(\operatorname{Fix}(A, G, \mu) \otimes C_{r}^{*}(G)\right)$. Since $W_{\nu * \mu} \vee$ is a corepresentation commuting with $W_{\mu}$, and $\operatorname{Ad} W_{\nu * \mu^{\vee}} \circ \operatorname{Ad} W_{\mu}=\operatorname{Ad} W_{\nu}$ by Proposition 2.3, $W_{\nu * \mu}$ is an $\operatorname{Ad} W_{\mu}$ cocycle implementing an exterior equivalence with $\operatorname{Ad} W_{\nu}$.

The uniqueness clause of [Qui2, Theorem 3.3] can be stated as a partial converse to Proposition 1.3:

Proposition 2.10. Nondegenerate cosystems $(B, G, \delta)$ and $(C, G, \epsilon)$ are conjugate if and only if there is a conjugacy $\Phi:\left(B \ltimes_{\delta} G, G\right) \rightarrow\left(C \ltimes_{\epsilon} G, G\right)$ such that

$$
\Phi \circ j_{G}^{\delta}=j_{G}^{\epsilon} \text {. }
$$

In fact, any such $\Phi$ restricts to a conjugacy of $\left(j_{B}(B), G, \operatorname{Ad}_{G}^{\delta} \otimes l\left(W_{G}\right)\right)$ with $\left(j_{C}(C), G, \operatorname{Ad}^{\epsilon} \otimes l\left(W_{G}\right)\right)$.

Proof. By Proposition 1.3, only the sufficiency requires proof. It follows from Proposition 1.4 that if $\Phi$ is a conjugacy as in the statement of the present theorem, then

$$
\Phi\left(\operatorname{Fix}\left(B \ltimes_{\delta} G, G, j_{G}^{\delta}\right)\right)=\operatorname{Fix}\left(C \ltimes_{\epsilon} G, G, j_{G}^{\epsilon}\right) .
$$

It follows from the uniqueness clause of [Qui2, Theorem 3.3] that $j_{B}(B)=$ $\operatorname{Fix}\left(B \ltimes G, G, j_{G}^{\delta}\right)$, and similarly for $\epsilon$, so $\Phi$ conjugates $\left(j_{B}(B), G, \operatorname{Ad}_{G}^{\delta} \otimes\right.$ $\left.\imath\left(W_{G}\right)\right)$ to $\left(j_{C}(C), G, \operatorname{Ad} j_{G}^{\epsilon} \otimes l\left(W_{G}\right)\right)$, whence $j_{C}^{-1} \circ \Phi \circ j_{B}$ conjugates $(B, G, \delta)$ to $(C, G, \epsilon)$.

\section{INDUCED $C^{*}$-ALGEBRAS AND ECHTERHOFF'S THEOREM}

Let $H$ be a closed subgroup of $G$, and let $(A, H)$ be a system. The induced $C^{*}$-algebra $\operatorname{Ind}_{H}^{G} A$ (or Ind $A$ if $G$ and $H$ are understood) consists of the continuous maps $x: G \rightarrow A$ such that $x(s h)=h^{-1} \cdot x(s)$ for all $s \in G, h \in H$ and the map $s H \mapsto\|x(s)\|$ vanishes at infinity on $G / H$. The induced action of $G$ on Ind $A$ is by left translation:

$$
(s \cdot x)(t)=x\left(s^{-1} t\right), \quad x \in \operatorname{Ind} A, s, t \in G .
$$

The following proposition shows that Ind $A$ is the result of an averaging process. Let $A \otimes C_{0}(G)$ carry the product $H$-action:

$$
h \cdot(a \otimes f)=h \cdot a \otimes h \cdot f,
$$

and define an $H$-equivariant nondegenerate homomorphism $\mu: C_{0}(G) \rightarrow$ $M\left(A \otimes C_{0}(G)\right)$ by $\mu(f)=1 \otimes f$.

Proposition 3.1. $\operatorname{Ind}_{H}^{G} A=\operatorname{Fix}\left(A \otimes C_{0}(G), H, \mu\right)$.

Proof. For ease of writing let

$$
X=A \otimes C_{0}(G), \quad Y=\mu\left(C_{c}(G)\right), \quad B=\operatorname{Fix}(X, H, Y)=\operatorname{Fix}(X, H, \mu) .
$$


We first observe that $Y X Y=C_{c}(G, A)$. To see this, first suppose that $x \in X$, $y, z \in Y$. Then for $s \in G$ we have

$$
(y x z)(s)=y(s) x(s) z(s),
$$

which vanishes for $s \notin \operatorname{supp} y$, so $y x z$ has compact support, hence is an element of $C_{c}(G, A)$. On the other hand, for $x \in C_{c}(G, A)$ we may choose $f \in C_{c}(G)$ which is identically 1 on $\operatorname{supp} x$, and then

$$
x=(1 \otimes f) x(1 \otimes f) \in Y X Y .
$$

We next observe that if $x \in C_{c}(G, A)$ then $E_{H} x \in C(G, A)$ and $s H \mapsto$ $\|E(x)(s)\|$ has compact support in $G / H$. For the first, note that

$$
E(x)(s)=\int_{H} h \cdot(x(s h)) d h,
$$

and the integrand is in $C_{c}(G, A)$, so $E x$ takes values in $A$. The uniform continuity of $x$ implies that $E x$ is norm continuous. For the other part, note that $x(\operatorname{sh})=0$ for $s \notin(\operatorname{supp} x) h^{-1}$, so $E(x)(s)=0$ for $s \notin(\operatorname{supp} x) H$, a compact subset of $G / H$.

We see from the above that $B \subset$ Ind $A$. To finish, we need only show that $B$ is dense in Ind $A$, which will follow from a partition of unity argument if we show that for each $s \in G\{x(s) \mid x \in B\}$ is dense in $A$ and $\left(1 \otimes C_{c}(G / H)\right) B \subset$ $B$ (e.g., [Ech, Lemma]). If $a \in A$ and $\epsilon>0$, choose a neighborhood $U$ of $e$ such that $\|a-h \cdot a\|<\epsilon$ for all $h \in U$. Further choose $f \in C_{c}(G)$ such that $f \geq 0, f(s h)=0$ for $h \notin U$, and $\int f(s h) d h=1$. Then

$$
\begin{aligned}
\|a-E(a \otimes f)(s)\| & =\left\|\int a f(s h) d h-\int h \cdot a f(s h) d h\right\| \\
& \leq \int\|a-h \cdot a\| f(s h) d h<\epsilon .
\end{aligned}
$$

That $B$ is closed under multiplication by $1 \otimes C_{c}(G / H)$ follows from the formula $(1 \otimes f) E(x)=E((1 \otimes f) x)=E(x)(1 \otimes f), \quad f \in C_{c}(G / H), x \in Y X Y$.

Corollary 3.2. Let $(A, H)$ be a system, and let $H$ be a closed subgroup of $G$. Then

$M($ Ind $A)=\left\{x \in M\left(A \otimes C_{0}(G)\right) \mid x(s h)=h^{-1} \cdot x(s)\right.$ for all $s \in G$ and $\left.h \in H\right\}$.

Moreover, if $\mu$ is a nondegenerate homomorphism of $B$ to $M\left(A \otimes C_{0}(G)\right)$ with $\mu(b)(s h)=h^{-1} \cdot \mu(b)(s)$ for all $b \in B, s \in G$, and $h \in H$, then $\mu$ is $a$ nondegenerate homomorphism to $M(\operatorname{Ind} A)$.

Proof. This follows immediately from Proposition 3.1, Lemma 1.7, and Corollary 1.8 .

Using the above corollary together with the nondegeneracy of Ind $A$ in $M\left(A \otimes C_{0}(G)\right)$ (which follows from a standard compactness argument), one readily checks that an $H$-equivariant nondegenerate homomorphism $\theta: A \rightarrow$ $M(B)$ induces a $G$-equivariant nondegenerate homomorphism, which we denote by Ind $\theta$, from Ind $A$ to $M(\operatorname{Ind} B)$.

We will need a reformulation of Echterhoff's characterization of induced systems [Ech, Theorem] which includes a uniqueness clause not explicitly stated by Echterhoff. We begin with a lemma. 
Lemma 3.3. Let $G$ act on both a $C^{*}$-algebra $A$ and a locally compact Hausdorff space $X$. Then there is a continuous G-equivariant map from $\operatorname{Prim} A$ to $X$ if and only if there is a G-equivariant nondegenerate homomorphism of $C_{0}(X)$ into $Z M(A)$.

Proof. By the Dauns-Hofmann theorem there is an isomorphism $\theta$ of $C_{b}(\operatorname{Prim} A)$ onto $Z M(A)$ such that

$$
\theta(f) a-f(I) a \in I, \quad f \in C_{b}(\operatorname{Prim} A), a \in A, I \in \operatorname{Prim} A,
$$

and $\theta$ is $G$-equivariant because the $G$-action on $\operatorname{Prim} A$ is given by $s \cdot I=\{s$. $a \mid a \in I\}$. Now given a continuous $G$-equivariant map $\phi: \operatorname{Prim} A \rightarrow X$, composing $\theta$ with the homomorphism $\phi^{*}: f \mapsto f \circ \phi$ of $C_{0}(X)$ into $C_{b}(\operatorname{Prim} A)$ gives a $G$-equivariant homomorphism $\theta \circ \phi^{*}$ of $C_{0}(X)$ into $Z M(A)$; we claim it is also nondegenerate. For if $a \in A$ and $\epsilon>0$, the set

$$
K=\{I \in \operatorname{Prim} a \mid\|a+I\| \geq \epsilon\}
$$

is compact in $\operatorname{Prim} A$, and hence so is $\phi(K)$. Then if $f \in C_{0}(X)$ satisfies $0 \leq f \leq 1$ and $\left.f\right|_{K} \equiv 1$, we have $\phi^{*}(f) \equiv 1$ on $K$, and

$$
\begin{aligned}
\left\|a-\theta \circ \phi^{*}(f) a\right\| & =\sup _{I \in \operatorname{Prim} A}\left\|a-\theta \circ \phi^{*}(f) a+I\right\| \\
& =\sup _{I \notin K}\|(1-f(\phi(I))) a+I\| \\
& \leq \sup _{I \notin K}\|a+I\| \leq \epsilon .
\end{aligned}
$$

Conversely, suppose $\mu: C_{0}(X) \rightarrow Z M(A)$ is nondegenerate and $G$-equivariant. Then each $I \in \operatorname{Prim} A$ gives a homomorphism $f \mapsto \theta^{-1}(\mu(f))(I)$ of $C_{0}(X)$, which is nonzero because $\mu$ is nondegenerate; and hence coincides with evaluation at some point $\phi(I)$ of $X$. The resulting map $\phi: \operatorname{Prim} A \rightarrow X$ is equivariant since $\mu$ is, and is continuous since each $\theta^{-1}(\mu(f))$ is.

Theorem 3.4 (Echterhoff). Let $(A, G)$ be a system, and let $H$ be a closed subgroup of $G$. Then there is a system $(B, H)$ such that $(A, G)$ is conjugate to $\left(\operatorname{Ind}_{H}^{G} B, G\right)$ if and only if there is a $G$-equivariant nondegenerate homomorphism $\mu: C_{0}(G / H) \rightarrow Z M(A)$. Indeed, if $I=\mu(K) A$, where $K=\{f \in$ $\left.C_{0}(G / H) \mid f(H)=0\right\}$, then we may take $B=A / I$, and $\Phi(a)(s)=s^{-1} \cdot a+I$ is a suitable conjugacy. Moreover, $(G, H)$ is unique up to conjugacy, in a sense made precise in Lemma 3.5 below.

Proof. Lemma 3.3 shows that our hypothesis is equivalent to Echterhoff's, so the first part follows from his theorem [Ech]. The uniqueness follows from a simple lemma, which we shall need again later:

Lemma 3.5. Suppose $\Psi$ is a conjugacy of (Ind $B_{1}, G$ ) and (Ind $B_{2}, G$ ) satisfying $\Psi(1 \otimes f)=1 \otimes f$ for $f \in C_{0}(G / H)$. Then there is a conjugacy $\theta$ of $\left(B_{1}, H\right)$ and $\left(B_{2}, H\right)$ such that $\Psi=$ Ind $\theta$. Further, if $\Psi$ already had the form Ind $\phi$ for some equivariant homomorphism $\phi: B_{1} \rightarrow B_{2}$, then $\theta=\phi$, and $\phi$ is actually an isomorphism.

Proof. Because we can approximate any element $x$ of $J_{1}=\left\{x \in\right.$ Ind $B_{1} \mid$ $x(e)=0\}$ by one of the form $f x$ with $f(H)=0$, the condition $\Psi(1 \otimes f)=$ $1 \otimes f$ implies that $\Psi$ maps $J_{1}$ onto the corresponding ideal $J_{2}$ of Ind $B_{2}$. Since $\epsilon_{e}: x \mapsto x(e)$ factors through an $H$-equivariant isomorphism of (Ind $\left.B_{i}\right) / J_{i}$ 
onto $B_{i}$, and $\Psi$ is $H$-equivariant, the unique isomorphism $\theta$ of $B_{1}$ onto $B_{2}$ satisfying $\theta \circ \epsilon_{e}=\epsilon_{e} \circ \Psi$ is a conjugacy. Finally, we check that $\Psi=\operatorname{Ind} \theta$ :

Ind $\theta(f)(s)=\theta(f(s))=\theta(f \cdot s(e))=\Psi(f \cdot s)(e)=(\Psi(f) \cdot s)(e)=\Psi(f)(s)$.

For the last observation, note that

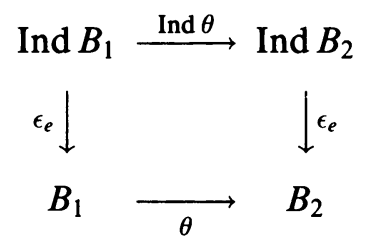

commutes.

\section{INDUCED SYSTEMS AND TWISTED COACTIONS}

Let $(B, G, \delta)$ be a cosystem, let $N$ be an amenable closed normal subgroup of $G$, and let $\lambda_{G, N}: C_{r}^{*}(G) \rightarrow C_{r}^{*}(G / N)$ be as in Section 2. The composition $\delta \mid=\left(l \otimes \lambda_{G, N}\right) \circ \delta$ is a coaction of $G / N$ on $B$, nondegenerate if $\delta$ is, called the restricted coaction [Man]. If $(\pi, \mu)$ is a covariant representation of $(B, G, \delta)$, then $(\pi, \mu \mid)$ is a covariant representation of the restricted cosystem $(B, G / N, \delta \mid)$ (recall that we let $\mu \mid$ denote the restriction of $\mu$ to $\left.C_{0}(G / N)\right)$. Moreover, $\left(j_{B} \times j_{G}\left|\left(B \ltimes_{\delta \mid} G / N\right), j_{B}, j_{G}\right|\right)$ is a cocrossed product for $(B, G / N, \delta \mid)$ [Man, Proposition 7], so that we may identify $j_{G / N}$ with $j_{G} \mid$. We mention that this latter fact can also be deduced easily from [Qui2, Proposition 3.1] by observing that $j_{G} \mid$ is $G / N$-equivariant.

The following proposition, showing that $B \ltimes G / N$ can be located inside $M(B \ltimes G)$ via averaging, is largely a reformulation of results of Mansfield [Man]:

Proposition 4.1 (Mansfield). If $(B, G)$ is a nondegenerate cosystem, then $B \ltimes$ $G / N=\operatorname{Fix}\left(B \ltimes G, N, j_{G}\right)$.

Proof. We recall a bit of Mansfield's construction: for each compact subset $K$ of $G$ let

$$
\mathscr{C}_{K}=\overline{j_{B}\left(\delta_{A_{K}(G)}(B)\right) j_{G}\left(C_{K}(G)\right)}
$$

where $C_{K}(G)=\left\{f \in C_{c}(G) \mid \operatorname{supp} f \subset K\right\}$ and $A_{K}(G)=A(G) \cap C_{K}(G)$. Then let

$$
\mathscr{D}=\bigcup\left\{\mathscr{C}_{K} \mid K \subset G, K \text { compact }\right\} .
$$

This is equivalent to Mansfield's definition of the set $\mathscr{D}$ since for every compact subset $K$ of $G$ there exists $f \in A_{c}(G)$ which is identically 1 on $K$. Mansfield proves that $\mathscr{D}$ is a dense ${ }^{*}$-subalgebra of $B \ltimes G$ [Man, Theorem 12]. He then proves [Man, Proposition 16] that there is a linear map $\Phi: \mathscr{D} \rightarrow M(B \ltimes G)$ such that

$$
\Phi\left(j_{B}(b) j_{G}(f)\right)=j_{B}(b) j_{G}(\phi(f)), \quad b \in \delta_{A_{c}(G)}(B), f \in C_{c}(G),
$$

where

Moreover, for $a, b \in \mathscr{D}$ we have

$$
\phi(f)(t)=\int_{N} f(t n) d n .
$$

$$
\Phi(a) b=\int_{N}(n \cdot a) b d n
$$


the integral converging in norm [Man, Lemma 18], and $\Phi$ is norm continuous on each $\mathscr{C}_{K}$ [Man, Lemma 15]. To compare $\Phi$ with $E$, we need to use something smaller than $\mathscr{D}$ to ensure that we are in the domain of $E$. For each compact subset $K$ of $G$ define

$$
\mathscr{C}_{K}^{\prime}=j_{G}\left(C_{K}(G)\right) j_{B}\left(\delta_{A_{K}(G)}(B)\right) j_{G}\left(C_{K}(G)\right),
$$

and let

$$
\mathscr{D}^{\prime}=\bigcup\left\{\mathscr{C}_{K}^{\prime} \mid K \subset G, K \text { compact }\right\}=j_{G}\left(C_{c}(G)\right) j_{B}\left(\delta_{A_{c}(G)}(B)\right) j_{G}\left(C_{c}(G)\right) .
$$

By nondegeneracy of $j_{G}, \mathscr{C}_{K}^{\prime}$ is dense in $\mathscr{C}_{K}$.

We show that $\Phi$ and $E_{N}$ agree on $\mathscr{D}^{\prime}$ : let $a \in \mathscr{D}^{\prime}$ and $\omega \in(B \ltimes G)^{*}$. Choose $b \in B \ltimes G$ and $\phi \in(B \ltimes G)^{*}$ such that $\omega=b \cdot \phi$, and choose a sequence $\left\{b_{j}\right\}$ in $\mathscr{D}$ such that $\left\|b-b_{j}\right\| \rightarrow 0$. Then

$$
\begin{aligned}
\langle\Phi(a), \omega\rangle & =\langle\Phi(a) b, \phi\rangle=\lim _{j}\left\langle\Phi(a) b_{j}, \phi\right\rangle=\lim _{j} \int_{N}\left\langle(n \cdot a) b_{j}, \phi\right\rangle d n \\
& =\lim _{j} \int_{N}\left\langle n \cdot a, b_{j} \cdot \phi\right\rangle d n=\lim _{j}\left\langle E_{N} a, b_{j} \cdot \phi\right\rangle \\
& =\left\langle E_{N} a, b \cdot \phi\right\rangle=\left\langle E_{N} a, \omega\right\rangle .
\end{aligned}
$$

Now, Mansfield further shows [Man, Theorem 19] that $B \ltimes G / N=\overline{\Phi(\mathscr{D})}$. Hence, by nondegeneracy of $j_{G}$ and $\delta$ and the continuity properties of $\Phi$ and $E_{N}$ we have

$$
\begin{aligned}
B \ltimes G / N & =C^{*}(\Phi(\mathscr{D}))=C^{*}\left(\bigcup_{K} \Phi\left(\mathscr{C}_{K}\right)\right)=C^{*}\left(\bigcup_{K} \Phi\left(\mathscr{C}_{K}^{\prime}\right)\right)=C^{*}\left(\bigcup_{K} E_{N}\left(\mathscr{C}_{K}^{\prime}\right)\right) \\
& =C^{*}\left(E_{N}\left(\mathscr{D}^{\prime}\right)\right)=\operatorname{Fix}\left(j_{B}\left(\delta_{A_{c}(G)}(B)\right), N, j_{G}\right)=\operatorname{Fix}\left(j_{B}(B), N, j_{G}\right) \\
& =\operatorname{Fix}\left(B \ltimes G, N, j_{G}\right) . \quad
\end{aligned}
$$

We now review some definitions and results from [PR, Section 2]. Let $(B, G, \delta)$ be a cosystem, and let $N$ be an amenable closed normal subgroup of $G$. A twist for $\delta$ over $G / N$ is a nondegenerate homomorphism $j: C_{0}(G / N) \rightarrow$ $M(B)$ such that

(i) $\delta \mid$ is implemented by $j$ (i.e., $(l, j)$ is a covariant representation of $(B, G / N, \delta \mid))$

(ii) $\delta \circ j(f)=j(f) \otimes 1$ for $f \in C_{0}(G / N)$.

If such a $j$ exists we say that $\delta$ is twisted over $G / N,(\delta, j)$ is a twisted coaction of $(G, G / N)$ on $B$, and $(B, G, G / N, \delta, j)$ is a twisted cosystem. A conjugacy between twisted cosystems $(B, G, G / N, \delta, j)$ and $(C, G, G / N, \epsilon, k)$ is a conjugacy $\theta:(B, G, \delta) \rightarrow(C, G, \epsilon)$ which respects the twists in the sense that $\theta \circ j=k$. A covariant representation of $(B, G, G / N, \delta, j)$ is a covariant representation $(\pi, \mu)$ of $(B, G, \delta)$ which preserves the twist in the sense that $\pi \circ j=\mu \mid$. If every covariant representation of $(B, G, G / N)$ factors through a representation of $C^{*}(\pi, \mu)$, we call $\left(C^{*}(\pi, \mu), \pi, \mu\right)$ a twisted cocrossed product for $(B, G, G / N)$; again, all such are isomorphic, and we denote a generic one by $\left(B \ltimes_{\delta, j, G / N} G, k_{B}, k_{G}\right)$ or $B \ltimes_{G / N} G$. The kernel $I_{j}$ of the quotient map $k_{B} \times k_{G}: B \ltimes G \rightarrow B \ltimes_{G / N} G$ coincides with the intersection of the kernels of all $\pi \times \mu$ for covariant representations $(\pi, \mu)$ of $(B, G, G / N)$, and is called the twisting ideal. Under the dual action of $G$ on the (untwisted) 
cocrossed product, the normal subgroup $N$ leaves the twisting ideal invariant, so we get a dual action of $N$ on the twisted cocrossed product. When $(\pi, \mu)$ is a covariant representation of $(B, G, G / N)$, we let $\pi \times_{G / N} \mu$ denote the unique nondegenerate homomorphism of $B \ltimes_{G / N} G$ such that $\left(\pi \times_{G / N} \mu\right) \circ k_{B}=\pi$ and $\left(\pi \times_{G / N} \mu\right) \circ k_{G}=\mu$. The definition of twisted coaction given above is equivalent to the one given in [PR, Definition 2.1] using the unitary $W_{j}$. The following result locates the image of $B \ltimes_{\delta \mid} G / N$ in $M\left(B \ltimes_{G / N} G\right)$.

Lemma 4.2. $k_{B} \times k_{G} \mid\left(B \ltimes_{\delta \mid} G / N\right)=k_{B}(B)$.

Proof. Although this can be deduced from [PR, Example 2.13], we give an elementary proof:

$$
\begin{aligned}
k_{B} \times k_{G} \mid(B \ltimes G / N) & =k_{B} \times k_{G} \mid\left(\overline{j_{B}(B) j_{G / N}\left(C_{0}(G / N)\right.}\right)=\overline{k_{B}(B) k_{G}\left(C_{0}(G / N)\right)} \\
& =\overline{k_{B}(B) k_{B} \circ j\left(C_{0}(G / N)\right)}=k_{B}(B),
\end{aligned}
$$

by nondegeneracy of $j$.

Lemma 4.3. Let $(B, G, G / N, \delta, j)$ be a twisted cosystem, and let $(\pi, \mu)$ be a covariant representation of $(B, G, \delta)$. Then:

(i) $(\pi, \pi \circ j)$ is a covariant representation of $(B, G / N, \delta \mid)$;

(ii) $\mu$ and $\pi \circ j$ commute;

(iii) $\left.\mu\right|^{\vee} *(\pi \circ j)$ is a nondegenerate homomorphism of $C_{0}(G / N)$ to $Z M\left(C^{*}(\pi, \mu)\right)$.

Proof. (i) follows from applying $\pi \otimes l$ to both sides of the covariance identity for $(l, j)$.

For (ii), if $f \in A(G), g \in C_{0}(G / N)$ then

$$
\begin{aligned}
\mu(f) \pi \circ j(g) & =S_{f}\left(W_{\mu}\right) \pi \circ j(g)=S_{f}\left(W_{\mu}(\pi \circ j(g) \otimes 1)\right) \\
& =S_{f}\left((\pi \otimes \imath) \circ \delta(j(g)) W_{\mu}\right)=S_{f}\left((\pi \circ j(g) \otimes 1) W_{\mu}\right) \\
& =\pi \circ j(g) \mu(f) .
\end{aligned}
$$

For (iii), we need only observe that $\left.\mu\right|^{\vee} *(\pi \circ j)$ commutes with both $\pi$ and $\mu$. The second follows by a calculation using Corollary 2.5 , while for the first, if $f \in A(G / N), b \in B$, then

$$
\begin{aligned}
\left.\mu\right|^{\vee} *(\pi \circ j)(f) \pi(b) & =S_{f}\left(W_{\left.\mu\right|^{\vee}} W_{\pi \circ j}(\pi(b) \otimes 1)\right) \\
& =S_{f}\left(W_{\mu \mid}^{*}(\pi \otimes \imath) \circ \delta \mid(b) W_{\pi \circ j}\right) \\
& =S_{f}\left((\pi(b) \otimes 1) W_{\mu \mid}^{*} W_{\pi \circ j}\right), \quad \text { because }(\pi, \mu \mid) \text { is covariant } \\
& =\left.\pi(b) \mu\right|^{\vee} *(\pi \circ j)(f) . \quad \square
\end{aligned}
$$

Theorem 4.4. Let $(B, G, G / N, \delta, j)$ be a twisted cosystem. Then the map $\Phi$ defined by $\Phi(c)(s)=\hat{\delta}_{s}^{-1}(c)+I_{j}$ is a conjugacy of the dual system $(B \ltimes G, G)$ onto the induced system ( $\left.\operatorname{Ind}_{N}^{G} B \ltimes_{G / N} G, G\right)$. The conjugacy carries $\left.j_{G}\right|^{\vee} *\left(j_{B} \circ j\right.$ ) onto the embedding $f \mapsto 1 \otimes f$ of $C_{0}(G / N)$ into $M\left(\operatorname{Ind} B \ltimes_{G / N} G\right)$.

Proof. Applying Lemma 4.3 (iii) to $\left(j_{B}, j_{G}\right)$, we get a nondegenerate homomorphism $\kappa=\left.j_{G}\right|^{\vee} *\left(j_{B} \circ j\right): C_{0}(G / N) \rightarrow Z M(B \ltimes G)$. We show that $\kappa$ is $G$-equivariant for left translation on $C_{0}(G / N)$ : for $f, g \in C_{c}(G / N)$, and 
$t \in G$ we have

$$
\begin{aligned}
\kappa\left((f * g) \cdot t^{-1}\right) & =\kappa\left(f \cdot t^{-1} * g\right) \\
& =\int_{G} j_{G}^{\vee}\left(s \cdot f \cdot t^{-1}\right) j_{B} \circ j\left(g \cdot s^{-1}\right) d s, \quad \text { by Corollary } 2.5 \\
& =\int_{G} j_{G}\left(t \cdot f^{\vee} \cdot s^{-1}\right) j_{B} \circ j\left(g \cdot s^{-1}\right) d s \\
& =\int_{G} t \cdot j_{G}\left(f^{\vee} \cdot s^{-1}\right) j_{B} \circ j\left(g \cdot s^{-1}\right) d s \\
& =\int_{G} t \cdot\left(j_{G}\left(f^{\vee} \cdot s^{-1}\right) j_{B} \circ j\left(g \cdot s^{-1}\right)\right) d s \\
& =t \cdot \int_{G} j_{G}\left(f^{\vee} \cdot s^{-1}\right) j_{B} \circ j\left(g \cdot s^{-1}\right) d s \\
& =t \cdot \int_{G} j_{G}^{\vee}(s \cdot f) j_{B} \circ j\left(g \cdot s^{-1}\right) d s \\
& =t \cdot \kappa(f * g) .
\end{aligned}
$$

We can now apply Echterhoff's theorem (Theorem 3.4). Let $I=\kappa(K)(B \ltimes G)$, where $K=\left\{f \in C_{0}(G / N) \mid f(N)=0\right\}$, be the ideal of $B \ltimes G$ associated to the $G$-equivariant nondegenerate homomorphism $\kappa$, so that Theorem 3.4 gives a conjugacy between $(B \ltimes G, G)$ and $\left(\operatorname{Ind}_{N}^{G}(B \ltimes G / I), G\right)$. Therefore, we can finish the proof by showing that $I$ coincides with the twisting ideal $I_{j}$. Since

$$
\left.I=\bigcap \begin{array}{l|l}
\operatorname{ker} \pi \times \mu & \begin{array}{l}
(\pi, \mu) \text { is a covariant representation of }(B, G) \text { such } \\
\text { that }(\pi \times \mu) \circ \kappa(f)=f(N) 1 \text { for } f \in C_{0}(G / N)
\end{array}
\end{array}\right\}
$$

and

$$
I_{j}=\bigcap\{\operatorname{ker} \pi \times \mu \mid(\pi, \mu) \text { is a covariant representation of }(B, G, G / N)\},
$$

it suffices to show that a covariant representation $(\pi, \mu)$ of $(B, G)$ preserves the twist if and only if $(\pi \times \mu) \circ \kappa=\mu_{e}$, the trivial nondegenerate homomorphism. By definition, $(\pi, \mu)$ preserves the twist if and only if $\pi \circ j=\mu \mid$, which in turn is equivalent to

$$
\mu_{e}=\left.\mu\right|^{\vee} *(\pi \circ j)=(\pi \times \mu) \circ\left(\left.j_{G}\right|^{\vee} *\left(j_{B} \circ j\right)\right)=(\pi \times \mu) \circ \kappa,
$$

so the result follows.

Theorem 4.4 generalizes a result of Olesen and Pedersen [OP3, Theorem 2.4]. They prove that if $G$ is abelian and $(A, G)$ is a system which is twisted in the sense of [Gre] over a closed subgroup $H$, then the dual system $(A \rtimes$ $G, \hat{G}$ ) is conjugate to the system ( $\operatorname{Ind}_{H^{\perp}}^{\hat{G}} A \rtimes_{H} G, \hat{G}$ ) induced from the twisted crossed product $A \rtimes_{H} G$. By [PR, Remark 2.3] the twisted system $(A, G, H)$ corresponds to a twisted cosystem $\left(A, \hat{G}, \hat{G} / H^{\perp}\right)$, and we have

$$
\begin{aligned}
(A \rtimes G, \hat{G}) & =(A \ltimes \hat{G}, \hat{G}), \\
\left(A \rtimes_{H} G, H^{\perp}\right) & =\left(A \ltimes_{\hat{G} / H^{\perp}} \hat{G}, H^{\perp}\right),
\end{aligned}
$$

so Olesen and Pedersen's result follows immediately from Theorem 4.4 above.

Theorem 4.4 has a lot of interesting consequences. We first give two corollaries which we shall need later, and then four more which we hope are of general interest. 
Corollary 4.5. Let $N$ be an amenable closed normal subgroup of $G$, let $(B, N$, $\epsilon)$ be a cosystem, and let $\delta=(l \otimes C) \circ \epsilon$ be the corresponding coaction of $G$ on $B$, where $C$ denotes the canonical nondegenerate homomorphism of $C_{r}^{*}(N)$ to $M\left(C_{r}^{*}(G)\right)$. Then $\left(B \ltimes_{\delta} G, G\right)$ is conjugate to ( $\left.\operatorname{Ind}_{N}^{G} B \ltimes_{\epsilon} N, G\right)$.

Proof. From [PR, Examples 2.4 and 2.14] we know that $\delta$ is indeed a coaction of $G$ on $B$ which is twisted over $G / N$ (by the trivial nondegenerate homomorphism of $\left.C_{0}(G / N)\right)$ and that $B \ltimes_{G / N} G$ is isomorphic to $B \ltimes N$. We show this isomorphism $\phi$ is $N$-equivariant, hence induces a conjugacy Ind $\phi$ of the induced systems, so that Theorem 4.4 gives the result.

Since $k_{G}$ is trivial on $C_{0}(G / N)$, it factors through the quotient map $C_{0}(G) \rightarrow$ $C_{0}(N)$ to give a nondegenerate homomorphism $j_{N}: C_{0}(N) \rightarrow B \ltimes_{G / N} G$, and $\left(k_{B}, j_{N}\right)$ is a covariant representation of $(B, N)$ with $C^{*}\left(k_{B}, j_{N}\right)=B \ltimes_{G / N} G$; $k_{B} \times j_{N}$ is the isomorphism found in [PR]. But since the quotient map and $k_{G}$ are both $N$-equivariant, so is $j_{N}$, and the equivariance of $k_{B} \times j_{N}$ follows.

Corollary 4.6. A nondegenerate cosystem $(B, G, \delta)$ is twisted over $G / N$ if and only if there is a G-equivariant nondegenerate homomorphism $\kappa: C_{0}(G / N) \rightarrow$ $Z M(B \ltimes G)$, and then $j_{G} \mid * \kappa^{\vee}$ is a twist for the coaction $\operatorname{Ad} W_{j_{G}}$ on $j_{B}(B)$. In particular, $\delta$ is unitary if and only if there is a G-equivariant nondegenerate homomorphism of $C_{0}(G)$ to $Z M(B \ltimes G)$.

Proof. If $\delta$ is twisted over $G / N$, then $(B \ltimes G, G)=\left(\operatorname{Ind}_{N}^{G} B \ltimes_{G / N} G, G\right)$ by Theorem 4.4 , so there exists a suitable $G$-equivariant nondegenerate homomorphism.

Conversely, let $\kappa: C_{0}(G / N) \rightarrow Z M(B \ltimes G)$ be a $G$-equivariant nondegenerate homomorphism. Since the cosystems $(B, G, \delta)$ and $\left(j_{B}(B), G, \operatorname{Ad} W_{j_{G}}\right)$ are conjugate, it is enough to show $j_{G} \mid * \kappa^{\vee}$ is a twist for $\operatorname{Ad} W_{j_{G}}$. To see that $j_{G} \mid * \kappa^{\vee}$ is a nondegenerate homomorphism of $C_{0}(G / N)$ to $M\left(j_{B}(B)\right)$, by Lemma 1.7 and [Qui2, Theorem 3.3] it suffices to show $j_{G} \mid * \kappa^{\vee}\left(C_{0}(G / N)\right)$ is nondegenerate in $M(B \ltimes G)$, commutes with $j_{G}\left(C_{0}(G)\right)$, and is pointwise fixed by $\hat{\delta}$. The only nonobvious point is the last, and it is enough to verify this for elements of the form $j_{G} \mid * \kappa^{\vee}(f * g)$ with $f, g \in C_{c}(G / N)$ : for $s \in G$ we have

$$
\begin{aligned}
\hat{\delta}_{s}\left(j_{G} \mid * \kappa^{\vee}(f * g)\right) & =\hat{\delta}_{s}\left(\int_{G / N} j_{G} \mid(t N \cdot f) \kappa^{\vee}\left(g \cdot t^{-1} N\right) d t N\right), \quad \text { by Corollary } 2.5 \\
& =\int_{G / N} j_{G} \mid(s t N \cdot f) \hat{\delta}_{s} \circ \kappa\left(\left(g \cdot t^{-1} N\right)^{\vee}\right) d t N \\
& =\int_{G / N} j_{G} \mid(s t N \cdot f) \kappa\left(s t N \cdot g^{\vee}\right) d t N \\
& =\int_{G / N} j_{G} \mid(t N \cdot f) \kappa\left(t N \cdot g^{\vee}\right) d t N \\
& =j_{G} \mid * \kappa^{\vee}(f * g) .
\end{aligned}
$$

By centrality, we have

$$
\operatorname{Ad} W_{j_{G} \mid}=\operatorname{Ad} W_{j_{G} \mid} \operatorname{Ad} W_{\kappa^{\vee}}=\operatorname{Ad} W_{j_{G} \mid * \kappa^{\vee}},
$$

so $j_{G} \mid * \kappa^{\vee}$ implements the restricted coaction $\operatorname{Ad} W_{j_{G}} \mid$. Since $j_{G}$ commutes with $j_{G} \mid * \kappa^{\vee}$, the coaction $\mathrm{Ad} W_{j_{G}}$ is trivial on the range of $j_{G} \mid * \kappa^{\vee}$. Therefore, $j_{G} \mid * \kappa^{\vee}$ is a twist for the cosystem $\left(j_{B}(B), G, \operatorname{Ad} W_{j_{G}}\right)$. 
The second statement is just the special case $N=\{e\}$.

Recall [QS, Definition 2.2] that a system $(A, G)$ is called regular if the regular representation is faithful, equivalently, the natural quotient map $A \rtimes G \rightarrow A \rtimes_{r} G$ is faithful.

Corollary 4.7. If $(B, G)$ is a cosystem which is twisted over $G / N$ for some amenable closed normal subgroup $N$ of $G$, then the dual system $(B \ltimes G, G)$ is regular.

Proof. Since $N$ is amenable, the system $\left(B \ltimes_{G / N} G, N\right)$ is regular. It follows from a result of Green [Gre, Theorem 17] that the crossed products $B \ltimes_{G / N} G \rtimes N$ and (Ind $\left.B \ltimes_{G / N} G\right) \rtimes G$ are strongly Morita equivalent. By [Rae2, Special Case 1.5], this Morita equivalence fits into the framework of the symmetric imprimitivity theorem of [Rae2, Theorem 1.1]. Hence, by [QS, Theorem 4.2], the system (Ind $B \ltimes_{G / N} G, G$ ) is also regular. The result now follows from Theorem 4.4.

The above results allow us to give an alternative proof of [PR, Theorem 4.1]:

Corollary 4.8. If $(B, G, G / N)$ is a nondegenerate twisted cosystem, then the dual crossed product $\left(B \ltimes_{G / N} G\right) \rtimes N$ is strongly Morita equivalent to $B$.

Proof. $\left(B \ltimes_{G / N} G\right) \rtimes N$ is strongly Morita equivalent to the induced system $\left(\right.$ Ind $\left._{N}^{G} B \ltimes_{G / N} G\right) \rtimes G$, hence by Theorem 4.4 to $(B \ltimes G) \rtimes G$. By Corollary 4.7 the latter crossed product is reduced. Therefore, Katayama's duality theorem applies, and $(B \ltimes G) \rtimes G$ is isomorphic to $B \otimes \mathscr{K}$. We conclude that $\left(B \ltimes_{G / N}\right.$ $G) \rtimes N$ is strongly Morita equivalent to $B \otimes \mathscr{K}$, hence to $B$.

We remark that nondegeneracy should be added as a hypothesis in [PR, Theorem 4.1], since its proof appeals to Mansfield's imprimitivity theorem [Man, Theorem 28], which uses nondegeneracy. On the other hand, it is tempting to conjecture that coactions twisted over $G / N$ are automatically nondegenerate, since they should behave like coactions of the amenable group $N$ (see [PR, Section 5(b)]).

Corollary 4.9. Let $(B, G, G / N)$ be a twisted cosystem, and let $\left(C_{0}(G) \otimes\left(B \ltimes_{G / N}\right.\right.$ $G), N$ ) be the product system (where $N$ acts by right translation on $C_{0}(G)$ and by the dual action on $\left.B \ltimes_{G / N} G\right)$. Then $B \ltimes G$ is strongly Morita equivalent to $\left(C_{0}(G) \otimes\left(B \ltimes_{G / N} G\right)\right) \rtimes N$.

Proof. Modulo a switch from left to right translation, $\operatorname{Ind}_{N}^{G} B \times_{G / N} G$ is the substitute for the fixed-point algebra for the product action on $C_{0}(G) \otimes\left(B \ltimes_{G / N}\right.$ $G$ ) used in [RW, Section 2], there denoted $G C\left(G, B \ltimes_{G / N} G\right)^{\alpha}$ ( $\alpha$ being the product action). By [RW, Theorem 2.2] $\operatorname{Ind}_{N}^{G} B \ltimes_{G / N} G$ is strongly Morita equivalent to $\left(C_{0}(G) \otimes\left(B \ltimes_{G / N} G\right)\right) \rtimes N$. The result follows from this and Theorem 4.4.

The following result partially generalizes [Qui2, Proposition 3.1] to twisted cosystems.

Corollary 4.10. If $(\pi, \mu)$ is a covariant representation of a twisted cosystem $(B, G, G / N)$ in $M(C)$, then $\pi \times_{G / N} \mu$ is faithful if and only if $\pi$ is faithful and there is an action of $N$ on the range of $\pi \times_{G / N} \mu$ such that $\mu$ is $N$-equivariant. 
Proof. Only the sufficiency requires proof. Let $\pi$ be faithful, and let $N$ act on $\pi \times_{G / N} \mu\left(B \ltimes_{G / N} G\right)$ in such a way that $\mu: C_{0}(G) \rightarrow M\left(\pi \times_{G / N} \mu\left(B \ltimes_{G / N} G\right)\right)$ is $N$-equivariant. We want to show $\pi \times_{G / N} \mu$ is an isomorphism, and by Lemma 3.5 it is enough to show it for the induced homomorphism $\operatorname{Ind}\left(\pi \times_{G / N} \mu\right)$. Let $\Phi: B \ltimes G \rightarrow \operatorname{Ind}\left(B \ltimes_{G / N} G\right)$ be the isomorphism of Theorem 4.4. It suffices to show that Ind $\left(\pi \times_{G / N} \mu\right) \circ \Phi$ is faithful, and by [Qui2, Proposition 3.1] it further suffices to show fidelity of $\operatorname{Ind}\left(\pi \times_{G / N} \mu\right) \circ \Phi_{\circ} j_{B}$. But a short calculation shows that the latter map is just $\pi$, which is faithful by hypothesis.

\section{LANDSTAD DUALITY FOR TWISTED COACTIONS}

In this section we generalize Landstad duality for coactions [Qui2, Theorem 3.3] to twisted coactions. In the proof of [Qui2, Theorem 3.3], it was shown that if $(B, G, \delta)$ is a nondegenerate cosystem, then $j_{B}(B)$ can be recovered from the dual system $(B \ltimes G, G)$ and the $G$-equivariant homomorphism $j_{G}$ via averaging. We generalize this to twisted cosystems, characterizing $k_{B}(B)$ in $M\left(B \ltimes_{G / N} G\right)$ :

Proposition 5.1. If $(B, G, G / N)$ is a nondegenerate twisted cosystem, then $k_{B}(B)=\operatorname{Fix}\left(B \ltimes_{G / N} G, N, k_{G}\right)$.

Proof. By Lemma 4.2, Proposition 4.1, and Proposition 1.4 we have

$$
\begin{aligned}
k_{B}(B) & =k_{B} \times k_{G}(B \ltimes G / N)=k_{B} \times k_{G}\left(\operatorname{Fix}\left(B \ltimes G, N, j_{G}\right)\right) \\
& =\operatorname{Fix}\left(B \ltimes_{G / N} G, N, k_{G}\right) .
\end{aligned}
$$

Theorem 5.2. Let $N$ be an amenable closed normal subgroup of $G$, and let $(A, N)$ be a system. Then $(A, N)$ is of the form $\left(B \ltimes_{G / N} G, N\right)$ if and only if there is an $N$-equivariant nondegenerate homomorphism $\mu: C_{0}(G) \rightarrow M(A)$. In this situation the twisted cosystem $(B, G, G / N)$ may be chosen to be nondegenerate, and then it is uniquely determined up to conjugacy by the further requirement that $k_{G}=\mu$.

Proof. If $(A, N)=\left(B \ltimes_{G / N} G, N\right)$, we can take $\mu=k_{G}$.

Conversely, assume the existence of $\mu$. We apply Landstad duality to $\left(\operatorname{Ind}_{N}^{G} A, G\right)$ and the homomorphism $\tilde{\mu}: C_{0}(G) \rightarrow M(\operatorname{Ind} A)$ defined by

$$
\tilde{\mu}(f)(s)=\mu\left(s^{-1} \cdot f\right),
$$

which we claim is nondegenerate and $G$-equivariant. To see that $\tilde{\mu}$ is a nondegenerate homomorphism to $M(\operatorname{Ind} A)$, it is enough by Corollary 3.2 to check $N$-equivariance:

$$
\tilde{\mu}(f)(s n)=\mu\left((s n)^{-1} \cdot f\right)=\mu\left(n^{-1} s^{-1} \cdot f\right)=n^{-1} \cdot \mu\left(s^{-1} \cdot f\right)=n^{-1} \cdot(\tilde{\mu}(f)(s)) .
$$

For $G$-equivariance of $\tilde{\mu}$, let $s, t \in G$, and $f \in C_{0}(G)$, and compute:

$$
\tilde{\mu}(t \cdot f)(s)=\mu\left(s^{-1} t \cdot f\right)=\tilde{\mu}(f)\left(t^{-1} s\right)=(t \cdot \tilde{\mu}(f))(s) .
$$

We can now deduce from [Qui2, Theorem 3.3] the existence of a unique $C^{*}$ subalgebra $B$ of $M(\operatorname{Ind} A)$ such that $\tilde{\mu}$ implements a nondegenerate coaction $\delta$ on $B,($ Ind $A, l, \tilde{\mu})$ is a cocrossed product for $(B, G, \delta)$, and the $G$-action agrees with $\hat{\delta}$.

The next step is to show that $\delta$ is twisted over $G / N$. Let $\eta: f \mapsto 1 \otimes f$ be the canonical $G$-equivariant nondegenerate homomorphism of $C_{0}(G / N)$ (with 
left $G$-translation) to $Z M($ Ind $A)$. Then $\eta^{\vee}$ may be viewed as a $G$-equivariant nondegenerate homomorphism of $C_{0}(G / N)$ (with right $G$-translation) to $Z M(B \ltimes G)$, so $j=\tilde{\mu} \mid * \eta$ is a twist for $\delta$ over $G / N$ by Corollary 4.6. By Theorem 4.4 the dual system $(B \ltimes G, G)$ is conjugate to the induced system $\left(\operatorname{Ind}_{N}^{G} B \ltimes_{G / N} G, G\right)$, and the canonical map $f \mapsto 1 \otimes f$ of $C_{0}(G / N)$ to $Z M\left(\right.$ Ind $\left.B \ltimes_{G / N} G\right)$ corresponds to the map $\left.\tilde{\mu}\right|^{\vee} * j: C_{0}(G / N) \rightarrow Z M(\operatorname{Ind} A)$. The conjugacy of $(A, N)$ and $\left(B \ltimes_{G / N} G, N\right)$ now follows from the uniqueness clause in Echterhoff's theorem because

$$
\eta=\left.\tilde{\mu}\right|^{\vee} * \tilde{\mu}|* \eta=\tilde{\mu}|^{\vee} * j .
$$

This concludes the proof of existence of a nondegenerate twisted cosystem $(B, G, G / N, \delta, j)$ such that $(A, N)=\left(B \ltimes_{G / N} G, N\right)$. For the uniqueness, we prove that, if $(C, G, G / N, \epsilon, \ell)$ is another nondegenerate twisted cosystem and there is a conjugacy $\Phi:\left(B \ltimes_{G / N} G, N\right) \rightarrow\left(C \ltimes_{G / N} G, N\right)$ satisfying $\Phi \circ k_{G}^{\delta}=$ $k_{G}^{\epsilon}$, then the twisted cosystems $(B, G, G / N)$ and $(C, G, G / N)$ are conjugate. It follows from Propositions 1.4 and 5.1 that

$$
\Phi\left(k_{B}(B)\right)=\Phi\left(\operatorname{Fix}\left(B \ltimes_{G / N} G, N, k_{G}^{\delta}\right)\right)=\operatorname{Fix}\left(C \ltimes_{G / N} G, N, k_{G}^{\epsilon}\right)=k_{C}(C) .
$$

Hence, the composite map $k_{C}^{-1} \circ \Phi \circ k_{B}$ gives an isomorphism of $B$ to $C$. We show that it conjugates the twisted coactions $(\delta, j)$ and $(\epsilon, \ell)$ : we have

$$
\begin{aligned}
\left(k_{C}^{-1} \circ \Phi \circ k_{B} \otimes \imath\right) \circ \delta & =\left(k_{C}^{-1} \circ \Phi \otimes \imath\right) \circ\left(k_{B} \otimes \imath\right) \circ \delta \\
& =\left(k_{C}^{-1} \otimes \imath\right) \circ(\Phi \otimes \imath) \circ \operatorname{Ad} W_{k_{G}^{\delta}} \circ\left(k_{B} \otimes 1\right) \\
& =\left(k_{C}^{-1} \otimes \imath\right) \circ \operatorname{Ad} W_{k_{G}^{\epsilon}}(\cdot \otimes 1) \circ \Phi \circ k_{B} \\
& =\epsilon \circ k_{C}^{-1} \circ \Phi \circ k_{B},
\end{aligned}
$$

and

$$
k_{C}^{-1} \circ \Phi \circ k_{B} \circ j=k_{C}^{-1} \circ \Phi \circ k_{G}^{\delta}\left|=k_{C}^{-1} \circ k_{G}^{\epsilon}\right|=k_{C}^{-1} \circ k_{C} \circ \ell=\ell .
$$

\section{APPlications of LANDSTAD DUALITY}

Our first application of Theorem 5.2 is a generalization of Proposition 2.9 to twisted cosystems:

Proposition 6.1. Let an amenable closed normal subgroup $N$ of $G$ act on $A$, and let $\mu, \nu: C_{0}(G) \rightarrow M(A)$ be commuting $N$-equivariant nondegenerate homomorphisms. Then $\operatorname{Fix}(A, N, \mu)=\operatorname{Fix}(A, N, \nu)$, and the (twisted) $G$-coactions (vouchsafed by Theorem 5.2) implemented by $\mu$ and $\nu$ on this common $C^{*}$ algebra are exterior equivalent.

Proof. The proof is an obvious modification of that of Proposition 2.9, with the $G$-actions replaced by $N$-actions.

Let $C: C_{r}^{*}(N) \rightarrow M\left(C_{r}^{*}(G)\right)$ denote the canonical nondegenerate homomorphism. As we discussed in the proof of Corollary 4.5, [PR, Examples 2.4 and 2.14] show that if $\epsilon$ is a coaction of an amenable closed normal subgroup $N$ of $G$ on $B$, then $(l \otimes C) \circ \epsilon$ is a coaction of $G$ on $B$ which is twisted over $G / N$ by the trivial nondegenerate homomorphism of $C_{0}(G / N)$, and moreover $B \ltimes_{G / N} G \cong B \ltimes N$. These results also follow from Theorem 5.2: 
Proposition 6.2. Let $\epsilon$ be a coaction of an amenable closed normal subgroup $N$ of $G$ on $B$. Then $(l \otimes C) \circ \epsilon$ is a nondegenerate coaction of $G$ on $B$ which is twisted over $G / N$ by the trivial nondegenerate homomorphism $\mu_{e}$ of $C_{0}(G / N)$. Moreover, the dual systems $(B \ltimes N, N)$ and $\left(B \ltimes_{G / N} G, N\right)$ are conjugate. Finally, a nondegenerate coaction of $G$ on $B$ is of this form if and only if its restriction to $G / N$ is trivial.

Proof. Let $R: C_{0}(G) \rightarrow C_{0}(N)$ be the restriction map. By Theorem 5.2, Lemma 1.6, and [Qui2, Theorem 3.3], $j_{N} \circ R$ implements a nondegenerate twisted coaction $\left(\operatorname{Ad} W_{j_{N} \circ R},\left(j_{N} \circ R\right) \mid\right)$ of $(G, G / N)$ on $\operatorname{Fix}\left(B \ltimes N, N, j_{N} \circ R\right)=$ $\operatorname{Fix}\left(B \ltimes N, N, j_{N}\right)=j_{B}(B)$, and moreover $(B \ltimes N, N)$ is conjugate to $\left(j_{B}(B) \ltimes_{G / N} G, N\right)$. The proof of the first two statements can be completed by showing that

$$
\left(j_{B}^{-1} \otimes l\right) \circ \operatorname{Ad} W_{j_{N} \circ R}(\cdot \otimes 1) \circ j_{B}=(l \otimes C) \circ \epsilon \quad \text { and } \quad j_{B}^{-1} \circ\left(j_{N} \circ R\right) \mid=\mu_{e} .
$$

The second is immediate from triviality of $R$ on $C_{0}(G / N)$, while the first follows from

$$
\begin{aligned}
\left(j_{B}^{-1}\right. & \otimes l) \circ \operatorname{Ad}\left(j_{N} \circ R \otimes l\left(W_{G}\right)\right)(\circ \otimes 1) \circ j_{B} \\
& =\left(j_{B}^{-1} \otimes l\right) \circ \operatorname{Ad}\left(j_{N} \otimes C\left(W_{N}\right)\right) \circ\left(j_{B} \otimes 1\right) \\
& =(l \otimes C) \circ\left(j_{B}^{-1} \otimes l\right) \circ \operatorname{Ad}\left(j_{N} \otimes l\left(W_{N}\right)\right) \circ\left(j_{B} \otimes 1\right) \\
& =(l \otimes C) \circ \epsilon .
\end{aligned}
$$

For the third statement, only the sufficiency requires proof, so assume that $\delta$ is a nondegenerate coaction of $G$ on $B$ such that $\delta \mid$ is trivial. Then the trivial nondegenerate homomorphism of $C_{0}(G / N)$ is a twist for $\delta$ over $G / N$. Since $k_{G}$ is trivial on $C_{0}(G / N)$, it factors as $k_{G}=\mu \circ R$ for some $N$-equivariant nondegenerate homomorphism $\mu: C_{0}(N) \rightarrow M\left(B \ltimes_{G / N} G\right)$. By [Qui2, Theorem 3.3], Lemma 1.6, and Proposition 5.1, $\mu$ implements a coaction $\epsilon$ of $N$ on $\operatorname{Fix}\left(B \ltimes_{G / N} G, N, \mu\right)=\operatorname{Fix}\left(B \ltimes_{G / N} G, N, k_{G}\right)=k_{B}(B)$. A calculation similar to the preceding paragraph shows that $\delta=\left(k_{B}^{-1} \otimes l\right) \circ \epsilon \circ k_{B}$.

Phillips and the second author ask in [PR, Section 5(b)] whether every twisted cocrossed product $B \ltimes_{G / N} G$ is isomorphic to an ordinary cocrossed product $B \ltimes N$ when $G$ splits as a semidirect product over $N$.

Proposition 6.3. Let $(B, G, G / N, \delta, j)$ be a nondegenerate twisted cosystem, and suppose that there is a continuous section $\sigma: G / N \rightarrow G$. Then there is a coaction $\epsilon$ of $N$ on $B$ such that $\delta$ is exterior equivalent to $(l \otimes C) \circ \epsilon$. In particular, the associated twisted cocrossed product is isomorphic to an ordinary cocrossed product by $N$.

Proof. Define a continuous map $\phi: G \rightarrow G$ by $\phi(s)=\sigma(s N)^{-1} s$, and then define a nondegenerate homomorphism $\mu: C_{0}(G) \rightarrow M\left(B \ltimes_{G / N} G\right)$ by

$$
\mu(f)=k_{G}(f \circ \phi) \text {. }
$$

Since $\phi$ commutes with right $N$-translation, $\mu$ is $N$-equivariant. Since $\mu$ commutes with $k_{G}$, by Proposition 6.1 the coactions $\operatorname{Ad} W_{\mu}$ and $\operatorname{Ad} W_{k_{G}}$ of $G$ on $k_{B}(B)$ are exterior equivalent. Since $f \circ \phi$ is constant for $f \in C_{0}(G / N), \mu \mid$ is scalar-valued, hence $\operatorname{Ad} W_{\mu} \mid=\operatorname{Ad} W_{\mu \mid}$ is trivial. Therefore, by Proposition 6.2 there is a coaction $\epsilon_{0}$ of $N$ on $k_{B}(B)$ such the coaction $\operatorname{Ad} W_{\mu}$ agrees 
with $(l \otimes C) \circ \epsilon$. But then $\epsilon=\left(k_{B}^{-1} \otimes \imath\right) \circ \epsilon_{0} \circ k_{B}$ is a coaction of $N$ on $B$, and $\delta=(l \otimes C) \circ \epsilon$.

As another application of Landstad duality, we give a shorter (but nonconstructive) proof of the decomposition theorem of Phillips and the second author [PR, Theorem 3.1] (under the penalty of a nondegeneracy hypothesis):

Proposition 6.4. Let $(B, G, \delta)$ be a nondegenerate cosystem. Then there is a nondegenerate twisted coaction of $(G, G / N)$ on $B \ltimes G / N$ such that

$$
B \ltimes G=(B \ltimes G / N) \ltimes_{G / N} G .
$$

Proof. Applying Theorem 5.2 with $A=B \ltimes G, \mu=j_{G}$, and the restriction to $N$ of the dual action $\hat{\delta}$, we see that $j_{G}$ implements a nondegenerate twisted coaction of $(G, G / N)$ on $C=\operatorname{Fix}\left(B \ltimes G, N, j_{G}\right)$ such that $B \ltimes G=C \ltimes_{G / N} G$. But $C=B \ltimes G / N$ by Proposition 4.1 .

\section{FULl COACTIONS}

Let $G$ be a locally compact group, $C^{*}(G)$ its full group $C^{*}$-algebra, and $i$ the canonical strictly continuous embedding of $G$ in $M\left(C^{*}(G)\right)$. We denote $i$ by $w_{G}$ when it is viewed as a unitary element of $M\left(C_{0}(G) \otimes C^{*}(G)\right)$. Note that $l \otimes \lambda_{G}\left(w_{G}\right)=W_{G}$. As usual, we identify $x \in C_{c}(G)$ with the element $\int x(s) i(s) d s$ of $C^{*}(G)$. Recall from [Rae1, Proposition 2(2)] that if $u: G \rightarrow U M(A)$ is a strictly continuous homomorphism, there is a unique nondegenerate homomorphism $u: C^{*}(G) \rightarrow M(A)$ such that $u(x)=\int x(s) u_{s} d s$ for $x \in C_{c}(G)$, called the integrated form of $u$. Here, the comultiplication $\delta_{G}$ on $C^{*}(G)$ will be the integrated form of the homomorphism $s \mapsto i(s) \otimes i(s)$ of $G$ into $U M\left(C^{*}(G) \otimes C^{*}(G)\right)$. As before, all $C^{*}$-tensor products are meant to be minimal unless otherwise specified.

Definition 7.1. A full coaction of $G$ on a $C^{*}$-algebra $A$ is a nondegenerate homomorphism $\delta: A \rightarrow M\left(A \otimes C^{*}(G)\right)$ such that

(a) $\delta(a)(1 \otimes x) \in A \otimes C^{*}(G)$, for $a \in A$ and $x \in C^{*}(G)$;

(b) $(\delta \otimes l) \circ \delta=\left(l \otimes \delta_{G}\right) \circ \delta$ as maps of $A$ into $M\left(A \otimes C^{*}(G) \otimes C^{*}(G)\right)$.

Examples 7.2. (1) The comultiplication $\delta_{G}$ is a full coaction of $G$ on $C^{*}(G)$. To see (a), note that if $x, y \in C_{c}(G)$ then

$$
\begin{aligned}
\delta_{G}(x)(1 \otimes y) & =\delta_{G}\left(\int x(s) i(s) d s\right)\left(\int y(t)(1 \otimes i(t)) d t\right) \\
& =\iint x(s) y(t)(i(s) \otimes i(s t)) d s d t \\
& =\iint x(s) y\left(s^{-1} t\right)(i(s) \otimes i(t)) d s d t .
\end{aligned}
$$

Approximating $F(s, t)=x(s) y\left(s^{-1} t\right)$ uniformly on supp $F$ by elements of $C_{c}(G) \odot C_{c}(G) \subset C_{c}(G \times G)$, and using, for example, [Rae1, Lemma 8], shows that this element of $M\left(C^{*}(G) \otimes C^{*}(G)\right)$ actually belongs to $C^{*}(G) \otimes C^{*}(G)$, giving (a). The coaction identity (b) holds because both sides are the integrated form of the homomorphism $s \mapsto i(s) \otimes i(s) \otimes i(s)$.

(2) The integrated form of the homomorphism $s \mapsto \lambda_{G}(s) \otimes i(s): G \rightarrow$ $U M\left(C_{r}^{*}(G) \otimes C^{*}(G)\right)$ factors through the regular representation $\lambda_{G}$ (because 
Ad $w_{G}\left(\lambda_{G}(s) \otimes i(s)\right)=\lambda_{G}(s) \otimes 1$ in $\left.M\left(\mathscr{K}\left(L^{2}(G)\right) \otimes C^{*}(G)\right)\right)$, and hence induces a nondegenerate homomorphism $\delta$ of $C_{r}^{*}(G)$ into $M\left(C_{r}^{*}(G) \otimes C^{*}(G)\right)$. Applying $\lambda_{G} \otimes l$ to the calculation in the previous example shows that $\delta$ is a full coaction of $G$ on $C_{r}^{*}(G)$.

(3) The usual modifications of the above arguments give full dual coactions $\hat{\alpha}$ of $G$ on any crossed product or reduced crossed product by an action $\alpha$ of $G ; \hat{\alpha}$ is characterised on generators by

$$
\hat{\alpha}\left(i_{A}(a)\right)=i_{A}(a) \otimes 1, \quad \hat{\alpha}\left(i_{G}(s)\right)=i_{G}(s) \otimes i(s) .
$$

Our main reason for using full coactions is to avoid hypotheses of amenability. In particular, we can restrict a full coaction of $G$ to a quotient $G / N$, even if the subgroup $N$ is not amenable, thus dodging some technical problems encountered in [Man], [PR]. To check the details, we need to consider the integrated form $q$ of the strictly continuous homomorphism $s \mapsto i(s N)$ of $G$ into $U M\left(C^{*}(G / N)\right)$. Let $x \in C_{c}(G)$. If we normalise Haar measures on $N, G$, and $G / N$ so that $\int_{G} x(s) d s=\int_{G / N} \int_{N} x(s n) d n d(s N)$, then

$$
\begin{aligned}
q(x) & =q\left(\int x(s) i(s) d s\right)=\int x(s) i(s N) d s \\
& =\int_{G / N}\left(\int_{N} x(s n) d n\right) i(s N) d(s N) ;
\end{aligned}
$$

in other words, $q(x)$ is the element of $C^{*}(G / N)$ given by the function $y(s N)=$ $\int x(s n) d n$ in $C_{c}(G / N)$. The map $x \mapsto y$ carries $C_{c}(G)$ onto $C_{c}(G / N)$ (given $y$, choose $\phi \in C_{c}(G)$ such that $\int \phi(\cdot n) d n \equiv 1$ on supp $y$, and let $x(s)=y(s N) \phi(s))$, and hence $q$ is actually a homomorphism of $C^{*}(G)$ onto $C^{*}(G / N)$.

Lemma 7.3. If $\delta: A \rightarrow M\left(A \otimes C^{*}(G)\right)$ is a full coaction, then $\delta \mid=(l \otimes q) \circ \delta$ is a full coaction of $G / N$ on $A$.

Proof. First of all, $\delta \mid$ is nondegenerate because $\delta$ and $q$ are. Definition 7.1 (a) follows from the corresponding property of $\delta$ and the surjectivity of $q$. To verify the coaction identity, note that

$$
\begin{aligned}
(\delta \mid \otimes l) \circ \delta \mid & =(l \otimes q \otimes l) \circ(\delta \otimes l) \circ(l \otimes q) \circ \delta \\
& =(l \otimes q \otimes q) \circ(\delta \otimes l) \circ \delta \\
& =(l \otimes q \otimes q) \circ\left(l \otimes \delta_{G}\right) \circ \delta,
\end{aligned}
$$

so we need $(q \otimes q) \circ \delta_{G}=\delta_{G / N} \circ q$. But both sides of this last equation are the integrated form of the homomorphism $s \mapsto i(s N) \otimes i(s N)$.

Definition 7.4. Let $\delta$ be a full coaction of $G$ on $A$. A covariant representation of $(A, G, \delta)$ is a pair $(\pi, \mu)$ of nondegenerate representations of $A, C_{0}(G)$ on $\mathscr{H}$ such that

$$
\pi \otimes l(\delta(a))=\mu \otimes l\left(w_{G}\right)(\pi(a) \otimes 1) \mu \otimes l\left(w_{G}^{*}\right) \quad \text { in } M\left(\mathscr{K}(\mathscr{H}) \otimes C^{*}(G)\right) .
$$

Definition 7.5. Let $\delta$ be a full coaction of $G$ on $A$. A cocrossed product for $(A, G, \delta)$ is a triple $\left(B, j_{A}, j_{G}\right)$ consisting of a $C^{*}$-algebra $B$ and nondegenerate homomorphisms $j_{A}: A \rightarrow M(B), j_{G}: C_{0}(G) \rightarrow M(B)$ satisfying

(a) $j_{A} \otimes l(\delta(a))=j_{G} \otimes l\left(w_{G}\right)\left(j_{A}(a) \otimes 1\right) j_{G} \otimes l\left(w_{G}^{*}\right)$ in $M\left(B \otimes C^{*}(G)\right)$; 
(b) for every covariant representation $(\pi, \mu)$ of $(A, G, \delta)$ there is a nondegenerate representation $\pi \times \mu$ of $B$ such that $(\pi \times \mu) \circ j_{A}=\pi$, $(\pi \times \mu) \circ j_{G}=\mu$

(c) the span of $\left\{j_{A}(a) j_{G}(f) \mid a \in A, f \in C_{0}(G)\right\}$ is a dense subspace of $B$.

Remarks 7.6. (1) Condition (a) implies that if $\rho$ is a nondegenerate representation of $B$, then $\left(\rho \circ j_{A}, \rho \circ j_{G}\right)$ is a covariant representation of $(A, G, \delta)$. Because we are using minimal tensor products, if $\rho$ is a faithful representation of $B$, then $\rho \otimes l$ is faithful on $B \otimes C^{*}(G)$, and the covariance of $\left(\rho \circ j_{A}, \rho \circ j_{G}\right)$ implies condition (a). Hence, modulo the change of tensor norm, this definition is equivalent to the one in [Rae3].

(2) As was pointed out in [Rae4] (see also [Quil]), the change to $\otimes_{\min }$ should make no difference to the arguments in [Rae4], and we claim that all the results of [Rae4] are valid for our present notion of full coaction: at worst, we have to apply the canonical quotient map $l \otimes_{\min } l$ to all the calculations. In particular, the argument of [Rae4, Theorem 2.13] shows that there is a unique cocrossed product, and we denote it by $\left(A \ltimes_{\delta} G, j_{A}, j_{G}\right)$.

(3) As observed in [Rae4, Remark 2.2(4)], composing a coaction satisfying the stronger conditions of [Rae4, Definition 2.1] with the quotient map $l \otimes_{\min } l$ onto $A \otimes_{\min } C^{*}(G)$ will give a full coaction as in Definition 7.1 ; in these cases, we are almost certainly throwing information away when we pass to $\otimes_{\min }$, although it is true that this information is irrelevant when we discuss representation theory [Rae4, Remark 2.5(1)] or take the cocrossed product [Rae4, Theorem 4.1]. Conversely, it is not clear whether every full coaction of the kind considered here is naturally associated with one of the kind studied in [Rae4]. Example 7.2 (2) suggests that one might have to change the algebra to do this: it seems unlikely that $C_{r}^{*}(G)$ carries a coaction of $G$ which is full in the sense of [Rae4]. To see the problem, note that the maximal tensor product $C^{*}(G) \otimes_{\max } C^{*}(G)$ has representations $\pi$ for which $\pi \circ \delta_{G}$ does not obviously factor through $C_{r}^{*}(G)$ : for example, how about the representation $\pi=\lambda_{G} \times \rho_{G}$ on $L^{2}(G)$ ? (Since the first draft of the present paper, the first author has verified that the natural coaction of $G$ on $C_{r}^{*}(G)$ does not factor through a full coaction in the sense of [Rae4].)

Definition 7.7. Let $\delta$ be a full coaction of $G$ on $A$. A twist for $\delta$ relative to $G / N$ is a nondegenerate homomorphism $j: C_{0}(G / N) \rightarrow M(A)$ such that

(1) $(l, j)$ is covariant in the sense that

$$
\delta \mid(a)=j \otimes l\left(w_{G / N}\right)(a \otimes 1) j \otimes l\left(w_{G / N}^{*}\right) \quad \text { in } M\left(A \otimes C^{*}(G / N)\right) ;
$$

(2) $\delta(j(f))=j(f) \otimes 1$ for $f \in C_{0}(G / N)$.

We refer to the pair $(\delta, j)$ as a twisted full coaction of $(G, G / N)$ on $A$.

We first observe that this definition can alternatively be couched in terms of the corepresentation $w=j \otimes l\left(w_{G / N}\right)$, as in [PR]:

Lemma 7.8. Let $j$ be a twist for $\delta$ relative to $G / N$, and let $w=j \otimes l\left(w_{G / N}\right) \in$ $M\left(A \otimes C^{*}(G / N)\right)$. Then

(a) $w_{12} w_{13}=l \otimes \delta_{G / N}(w)$;

(b) $\delta \mid(a)=w(a \otimes 1) w^{*}$

(c) $\delta \otimes l(w)=w_{13}$. 
Conversely, if $w \in M\left(A \otimes C^{*}(G / N)\right)$ is a unitary satisfying (a), (b), and (c), then there is a twist $j: C_{0}(G / N) \rightarrow M(A)$ for $\delta$ such that $j(f)=S_{f}(w)$ for $f \in B(G / N)$, and $w=j \otimes l\left(w_{G / N}\right)$.

Proof. By Lemma A.1 below, the nondegenerate homomorphism $j$ determines and is determined by the unitary $w=j \otimes l\left(w_{G / N}\right)$ satisfying (a). Equation (b) is a restatement of Definition 7.7 (1), and slicing (c) with elements of $A(G / N) \subset$ $C^{*}(G / N)^{*}$ gives Definition 7.7 (2). Conversely, given Definition 7.7 (2), we can deduce immediately that

$$
S_{f}(\delta \otimes l(w))=\delta\left(S_{f}(w)\right)=\delta(j(f))=j(f) \otimes 1=S_{f}\left(w_{13}\right)
$$

for all $f \in A(G / N)$. However, it follows from Lemma A.2 that $j(f)=S_{f}(w)$ for $f \in B(G / N)$. Since $\delta, j$, and $S_{f}$ are all strictly continuous, this allows us to extend the calculation (7.1) to $f \in B(G / N)$, which is enough to give (c).

Examples 7.9. (1) The lemma shows that if $G$ is amenable, this notion of twist is equivalent to the one in [PR]. In particular, when $G$ is abelian, a coaction $\delta$ of $G$ has a twist relative to $G / N$ if and only if the corresponding action of $\hat{G}$ has a Green twisting map on $N^{\perp}$ (cf. [PR, 2.3]).

(2) If $\epsilon$ is a full coaction of $N$, and $C: C^{*}(N) \rightarrow M\left(C^{*}(G)\right)$ is the integrated form of $n \mapsto i_{G}(n)$, then $\delta=(l \otimes C) \circ \epsilon$ is a full coaction of $G$, and $j(f)=f(N) 1$ is a twist for $\delta$ relative to $G / N$ (see the proof of Corollary 4.5).

(3) There is always a twisted full coaction of $(G, G / N)$ on the cocrossed product $A \ltimes_{\delta \mid} G / N$ :

Lemma 7.10. If $\delta$ is a full coaction of $G$ on $A$, there is a full coaction $\gamma$ of $G$ on the cocrossed product $A \ltimes_{\delta \mid} G / N$ such that

$$
\gamma\left(j_{A}(a) j_{G / N}(f)\right)=j_{A} \otimes l(\delta(a))\left(j_{G / N}(f) \otimes 1\right),
$$

and $j_{G / N}$ is a twist for $\gamma$ relative to $G / N$.

Proof. Let $\pi=\left(j_{A} \otimes \imath\right) \circ \delta, \mu=j_{G / N} \otimes \imath$. As in [PR, 3.3], the covariance of $\left(j_{A}, j_{G / N}\right)$ and the identity $\delta \otimes l(\delta \mid(a))=l \otimes \sigma(\delta \mid \otimes l(\delta(a)))$ imply that

$$
\pi \otimes l(\delta \mid(a)) \mu \otimes \imath\left(w_{G / N}\right)=\mu \otimes \imath\left(w_{G / N}\right)(\pi(a) \otimes 1) .
$$

The universal property of the cocrossed product $A \ltimes_{\delta \mid} G / N$ now gives us a nondegenerate homomorphism $\gamma=\pi \times \mu$ of $A \ltimes_{\delta \mid} G / N$ into $M\left(\left(A \ltimes_{\delta \mid} G / N\right) \otimes\right.$ $C^{*}(G)$ ) satisfying (7.2) (see Corollary 7.14 below); the proof that $\gamma$ is a coaction carries over from [PR, Lemma 3.3] (just replace $\lambda_{G}(z) \in C_{r}^{*}(G)$ by $z \in C^{*}(G)$ ). We can use the covariance of $\left(j_{A}, j_{G / N}\right)$ and the argument of [PR, Lemma 3.4] to verify that $j=j_{G / N}$ has property (1) of Definition 7.7; since equation (7.2) implies property (2), we deduce that $j$ is a twist for $\gamma$.

Definition 7.11. Let $(\delta, j)$ be a twisted full coaction of $(G, G / N)$ on $A$. We say that a covariant representation $(\pi, \mu)$ of $(A, G, \delta)$ preserves $j$ if $\pi \circ j=$ $\left.\mu\right|_{C_{0}(G / N) \text {. Let }}$

$$
I_{j}=\bigcap\{\operatorname{ker} \pi \times \mu \mid(\pi, \mu) \text { is covariant and preserves } j\} .
$$

The twisted cocrossed product $A \ltimes_{\delta, G / N, j} G$ is the quotient $A \ltimes_{\delta} G / I_{j}$. We write $q_{j}$ for the quotient map of $A \ltimes_{\delta} G$ onto $A \ltimes_{G / N} G$. 
Remark 7.12. Once we have established the analogue of [PR, Proposition 2.11] for twisted cocrossed products by full coactions (Proposition 7.13 below), the proof of [PR, Theorem 3.1] carries over almost verbatim; all we have to do is replace $l \otimes \lambda_{G}\left(w_{G}\right), l \otimes \lambda_{G, N}\left(w_{G / N}\right)$ by $w_{G}, w_{G / N}$ (and to make it easier, in the preprint half the $\lambda_{G}$ 's are already missing). Hence the decomposition

$$
A \ltimes_{\delta} G=\left(A \ltimes_{\delta \mid} G / N\right) \ltimes_{G / N} G
$$

is valid whether $N$ is amenable or not.

Proposition 7.13 (cf. [PR, Proposition 2.11]). Let $(\delta, j)$ be a twisted full coaction of $(G, G / N)$ on $A$, and let $k_{A}=q_{j} \circ j_{A}, k_{G}=q_{j} \circ j_{G}$. Then

(a) $k_{A} \otimes l(\delta(a))=k_{G} \otimes l\left(w_{G}\right)\left(k_{A}(a) \otimes 1\right) k_{G} \otimes l\left(w_{G}^{*}\right), \quad a \in A$;

(b) $k_{A} \circ j=k_{G} \mid C_{0}(G / N)$;

(c) for every covariant representation $(\pi, \mu)$ of $(A, G, \delta)$ which preserves $j$, there is a nondegenerate representation $\pi \times_{G / N} \mu$ of $A \ltimes_{\delta, G / N, j} G$ such that

$$
\left(\pi \times_{G / N} \mu\right) \circ k_{A}=\pi, \quad\left(\pi \times_{G / N} \mu\right) \circ k_{G}=\mu ;
$$

(d) the set $\left\{k_{A}(a) k_{G}(f) \mid a \in A, f \in C_{0}(G)\right\}$ spans a dense subspace of $A \ltimes_{G / N} G$.

Proof. Property (d) follows from the corresponding property of ( $A \ltimes_{\delta} G, j_{A}$, $j_{G}$ ), which is given in Definition 7.5 (c), and (a) from equation (a) of Definition 7.5. For (b), we have to show that

$$
q_{j}\left(j_{A} \circ j(f)-j_{G}(f)\right)=0, \quad f \in C_{0}(G / N),
$$

or, equivalently, that $j_{A} \circ j(f)=j_{G}(f)$ belongs to $\operatorname{ker} \phi \times \nu$ whenever $(\phi, \nu)$ preserves $j$. However,

$$
\phi \times \nu\left(j_{A} \circ j(f)-j_{G}(f)\right)=\phi \circ j(f)-\nu(f)
$$

vanishes precisely when $(\phi, \nu)$ preserves $j$, so this is clear. Finally, if $(\pi, \nu)$ preserves $j, \pi \times \nu$ vanishes on $I_{j}$, and hence factors through a nondegenerate representation $\phi$ of $A \ltimes_{G / N} G=A \ltimes G / I_{j}$-in other words, $\pi \times \mu=\phi \circ q_{j}$. But then

$$
\phi \circ k_{A}=\phi \circ q_{j} \circ j_{A}=(\pi \times \mu) \circ j_{A}=\pi, \quad \phi \circ k_{G}=\mu,
$$

and we can take $\pi \times_{G / N} \mu=j$.

Corollary 7.14. Suppose $(\delta, j)$ is a twisted full coaction of $(G, G / N)$ on $A$, and $\pi: A \rightarrow M(C), \mu: C_{0}(G) \rightarrow M(C)$ is a pair of nondegenerate homomorphisms such that

(a) $\pi \otimes l(\delta(a))=\mu \otimes \imath\left(w_{G}\right)(\pi(a) \otimes 1) \mu \otimes l\left(w_{G}^{*}\right) \quad$ in $M\left(C \otimes C^{*}(G)\right)$;

(b) $\pi \circ j=\left.\mu\right|_{C_{0}(G / N)}$.

Then there is a nondegenerate homomorphism $\pi \times_{G / N} \mu: A \ltimes_{G / N} G \rightarrow M(C)$ such that $\left(\pi \times_{G / N} \mu\right) \circ k_{A}=\pi$ and $\left(\pi \times_{G / N} \mu\right) \circ k_{G}=\mu$.

Proof. If we represent $C$ nondegenerately and faithfully on a Hilbert space $\mathscr{H}$, then $(\pi, \mu)$ becomes a covariant representation of $(A, G, \delta)$ which preserves $j$. Thus the proposition gives a nondegenerate representation $\pi \times_{G / N} \mu$ with $\left(\pi \times_{G / N} \mu\right) \circ k_{A}=\pi$ and $\left(\pi \times_{G / N} \mu\right) \circ k_{G}=\mu$. These equations imply that 
$\pi \times_{G / N} \mu$ takes values in $M(C)=\{T \in \mathscr{L}(\mathscr{H}) \mid T C \subset C$ and $C T \subset C\}$, and, together with the nondegeneracy of $\pi$ and $\mu$, that $\pi \times_{G / N} \mu$ is nondegenerate as a homomorphism into $M(C)$.

Corollary 7.15. The properties (a)-(d) of Proposition 7.13 uniquely characterize the triple $\left(A \ltimes_{G / N} G, k_{A}, k_{G}\right)$ up to isomorphism.

Finally, the proofs of Theorems 4.4 and 5.2 carry over to full coactions, without any assumption of amenability of $N$. The same holds true for their corollaries, except that we need amenability of $N$ in Corollary 4.7 and nondegeneracy of $\epsilon$ in Proposition 6.2.

\section{APPENDIX}

We establish an analog of Lemma 1.2 with $C^{*}(G)$ replacing $C_{r}^{*}(G)$ :

Lemma A.1. Suppose $\mu: C_{0}(G) \rightarrow M(A)$ is a nondegenerate homomorphism. Then $w=\mu \otimes \imath\left(w_{G}\right)$ is a unitary element of $M\left(A \otimes C^{*}(G)\right)$ satisfying

$$
w_{12} w_{13}=\imath \otimes \delta_{G}(w) .
$$

Conversely, if $w \in U M\left(A \otimes C^{*}(G)\right)$ satisfies (A.1), there is a nondegenerate homomorphism $\mu$ of $C_{0}(G)$ to $A$ such that

$$
\begin{gathered}
\mu(f)=S_{f}(w), \quad f \in A(G) \subset C^{*}(G)^{*} ; \\
w=\mu \otimes l\left(w_{G}\right) .
\end{gathered}
$$

Proof. Because $\mu$ is nondegenerate, $\mu \otimes l$ extends uniquely to a strictly continuous *-homomorphism of $M\left(C_{0}(G) \otimes C^{*}(G)\right)$, and the identity (A.1) follows from the equation $\left(w_{G}\right)_{12}\left(w_{G}\right)_{13}=\imath \otimes \delta_{G}\left(w_{G}\right)$ in $M\left(C_{0}(G) \otimes C^{*}(G) \otimes C^{*}(G)\right)$. So now suppose that $w$ satisfies (A.1). Since the functional on $C^{*}(G)$ determined by $f \in A(G) \subset B(G)=C^{*}(G)^{*}$ factors through $\lambda_{G}$, we have $S_{f}(w)=$ $S_{f}\left(l \otimes \lambda_{G}(w)\right)$, and we can apply Lemma 1.2 to $W=\imath \otimes \lambda_{G}(w)$ to deduce that $\mu(f)=S_{f}(w)$ defines a nondegenerate homomorphism $\mu$ of $C_{0}(G)$ into $M(A)$. It therefore remains to verify that $w=\mu \otimes l\left(w_{G}\right)$.

Note straightaway that for $f \in A(G)$, we have

$$
S_{f}(w)=\mu(f)=\mu\left(S_{f}\left(w_{G}\right)\right)=S_{f}\left(\mu \otimes \imath\left(w_{G}\right)\right),
$$

which implies $l \otimes \lambda_{G}(w)=\mu \otimes \lambda_{G}\left(w_{G}\right)$ because $A(G) \subset C_{r}^{*}(G)^{*} \subset C^{*}(G)^{*}$ separates points of $C_{r}^{*}(G)$; we need to show (A.2) also holds for $f \in B(G)$. The problem is that, while the nondegenerate homomorphism $\mu$ extends to a strictly continuous homomorphism $\bar{\mu}$ on $C_{b}(G)=M\left(C_{0}(G)\right)$, it is not obvious that we then have $\bar{\mu}(f)=S_{f}(w)$ for $f \in B(G)$, and in the last equality in (A.2) we need to use $\bar{\mu}$ rather than $\mu$. The next lemma fills this gap, and is also useful elsewhere.

Lemma A.2. Let $w \in U M\left(A \otimes C^{*}(G)\right)$ satisfy (A.1), and let $\mu: C_{0}(G) \rightarrow M(A)$ be the nondegenerate homomorphism such that $\mu(f)=S_{f}(w)$ for $f \in A(G)$. Let $\bar{\mu}$ be the strictly continuous extension of $\mu$ to $C_{b}(G)=M\left(C_{0}(G)\right)$. Then $\bar{\mu}(f)=S_{f}(w)$ for $f \in B(G)$.

Proof. We can certainly define a bounded linear map $\nu: B(G) \rightarrow M(A)$ by $\nu(f)=S_{f}(w)$. For $f, g \in B(G)$, the functional $(f \otimes g) \circ \delta_{G}$ is given by the pointwise product $f g \in B(G)$. (To see this, approximate $g$ by $x \cdot h$ for some 
$x \in C_{c}(G) \subset C^{*}(G)$, and compute $\left\langle f \otimes x \cdot h, \delta_{G}(y)\right\rangle=\left\langle f \otimes h, \delta_{G}(y)(1 \otimes x)\right\rangle$ for $y \in C_{c}(G)$.) Thus equation (A.1) implies

$$
\begin{aligned}
\nu(f) \nu(g) & =S_{f}(w) S_{g}(w)=S_{f \otimes g}\left(w_{12} w_{13}\right) \\
& =S_{f \otimes g}\left(l \otimes \delta_{G}(w)\right)=S_{(f \otimes g) \circ \delta_{G}}(w)=\nu(f g),
\end{aligned}
$$

and $\nu$ is a homomorphism. Now because $\mu$ is nondegenerate as a homomorphism of $A(G)$ into $M(A)$, the multipliers $\bar{\mu}(f)$ and $\nu(f)$ are determined by their values on elements of the form $\mu(g) a$ for $g \in A(G)$. Then because $\mu=\left.\nu\right|_{A(G)}$ and $A(G)$ is an ideal in $B(G)$, we have

$$
\nu(f)(\mu(g) a)=\nu(f)(\nu(g) a)=\nu(f g) a=\mu(f g) a=\bar{\mu}(f)(\mu(g) a) .
$$

This completes the proofs of Lemmas A.1 and A.2.

Remark. It follows from Lemma A.2 that $\nu(f)=S_{f}(w)$ actually defines a *homomorphism $\nu$ of $B(G)$ into $M(A)$. We saw in the proof how to see that $\nu(f) \nu(g)=\nu(f g)$ directly, but the identity $\nu(\bar{f})=\nu\left(f^{*}\right)$ may not be so easy: the proof given here depends on the corresponding property of $\mu=\left.\nu\right|_{A(G)}$, which is quite subtle (cf. [NT, proof of Theorem A.1]).

\section{REFERENCES}

[APT] C. A. Akemann, G. K. Pedersen, and J. Tomiyama, Multipliers of $C^{*}$-algebras, J. Funct. Anal. 13 (1973), 277-301.

[Ech] S. Echterhoff, On induced covariant systems, Proc. Amer. Math. Soc. 108 (1990), 703-706.

[Eym] P. Eymard, L'algèbre de Fourier d'un groupe localement compact, Bull. Soc. Math. France 92 (1964), 181-236.

[Gli] J. Glimm, Families of induced representations, Pacific J. Math. 12 (1962), 885-911.

[GL] E. C. Gootman and A. J. Lazar, Applications of non-commutative duality to crossed product $C^{*}$-algebras determined by an action or coaction, Proc. London Math. Soc. (3) 59 (1989), 593-624.

[Gre] P. Green, The local structure of twisted covariance algebras, Acta Math. 140 (1978), 191-250.

[IT] S. Imai and H.Takai, On a duality for $C^{*}$-crossed products by a locally compact group, J. Math. Soc. Japan 30 (1978), 495-504.

[Ior] V. Iorio, Hopf- $C^{*}$-algebras and locally compact groups, Pacific J. Math. 87 (1980), 75-96.

[Kat] Y. Katayama, Takesaki's duality for a non-degenerate co-action, Math. Scand. 55 (1985), 141-151.

[Lan1] M. B. Landstad, Duality for dual covariance algebras, Comm. Math. Phys. 52 (1977), 191-202.

[Lan2] _ Duality for dual $C^{*}$-covariance algebras over compact groups, unpublished manuscript, 1978.

[Lan3] _ Duality theory for covariant systems, Trans. Amer. Math. Soc. 248 (1979), 223-267.

[LPRS] M. B. Landstad, J. Phillips, I. Raeburn, and C. E. Sutherland, Representations of crossed products by coactions and principal bundles, Trans. Amer. Math. Soc. 299 (1987), 747-784.

[Man] K. Mansfield, Induced representations of crossed products by coactions, J. Funct. Anal. 97 (1991), 112-161.

[NT] Y. Nakagami and M. Takesaki, Duality for crossed products of von Neumann algebras, Lecture Notes in Math., vol. 731, Springer-Verlag, New York, 1979.

[OP] D. Olesen and G. K. Pedersen, Applications of the Connes spectrum to $C^{*}$-dynamical systems, J. Funct. Anal. 30 (1978), 179-197.

[OP2] _ Applications of the Connes spectrum to $C^{*}$-dynamical systems. II, J. Funct. Anal. 36 (1980), 18-32. 
[OP3] _ Partially inner $C^{*}$-dynamical systems, J. Funct. Anal. 66 (1986), 262-281.

[PaR] J. A. Packer and I. Raeburn, Twisted crossed products of $C^{*}$-algebras. II, Math. Ann. 289 (1990), 595-612.

[Ped] G. K. Pedersen, Dynamical systems and crossed products, Proc. Sympos. Pure Math., vol. 38, Amer. Math. Soc., Providence, R. I., 1982, pp. 271-283.

[PR] J. Phillips and I. Raeburn, Twisted crossed products by coactions, J. Austral. Math. Soc. Ser. A 56 (1994), 320-344.

[Qui1] J. C. Quigg, Full C*-crossed product duality, J. Austral. Math. Soc. Ser. A 50 (1991), 34-52.

[Qui2] _ Landstad duality for $C^{*}$-coactions, Math. Scand. 71 (1992), 277-294.

[QS] J. C. Quigg and J. Spielberg, Regularity and hyporegularity in $C^{*}$-dynamical systems, Houston J. Math. 18 (1992), 139-152.

[Rae1] I. Raeburn, On crossed products and Takai duality, Proc. Edinburgh Math. Soc. 31 (1988), 321-330.

[Rae2] - Induced $C^{*}$-algebras and a symmetric imprimitivity theorem, Math. Ann. 280 (1988), 369-387.

[Rae3] — A duality theorem for crossed products by nonabelian groups, Proc. Centre Math. Anal. Austral. Nat. Univ. 15 (1987), 214-227.

[Rae4] - On crossed products by coactions and their representation theory, Proc. London Math. Soc. (3) 64 (1992), 625-652.

[RR] I. Raeburn and J. Rosenberg, Crossed products of continuous-trace $C^{*}$-algebras by smooth actions, Trans. Amer. Math. Soc. 305 (1988), 1-45.

[RW] I. Raeburn and D. P. Williams, Pull-backs of $C^{*}$-algebras and crossed products by certain diagonal actions, Trans. Amer. Math. Soc. 287 (1985), 755-777.

[Tak] H. Takai, On a duality for crossed products of $C^{*}$-algebras, J. Funct. Anal. 19 (1975), 25-39.

[Val] J.-M. Vallin, $C^{*}$-algèbres de Hopf et $C^{*}$-algèbres de Kac, Proc. London Math. Soc. (3) 50 (1985), 131-174.

[Wor] S. L. Woronowicz, Pseudospaces, pseudogroups and Pontryagin duality, Proc. Internat. Conf. Math. Phys. (Lausanne 1979), Lecture Notes in Physics, vol. 116, Springer-Verlag, New York, 1980, pp. 407-412.

Department of Mathematics, Arizona State University, Tempe, Arizona 85287

E-mail address: quigg@asu.edu

Department of Mathematics, University of Newcastle, Newcastle, New South Wales 2308, Australia

E-mail address: iain@frey.newcastle.edu.au 